Review

\title{
Conformal Symmetry in Field Theory and in Quantum Gravity
}

\author{
Lesław Rachwał \\ Instituto de Física, Universidade de Brasília, Brasília DF 70910-900, Brazil; grzerach@gmail.com
}

Received: 29 August 2018; Accepted: 9 November 2018; Published: 15 November 2018

\begin{abstract}
Conformal symmetry always played an important role in field theory (both quantum and classical) and in gravity. We present construction of quantum conformal gravity and discuss its features regarding scattering amplitudes and quantum effective action. First, the long and complicated story of UV-divergences is recalled. With the development of UV-finite higher derivative (or non-local) gravitational theory, all problems with infinities and spacetime singularities might be completely solved. Moreover, the non-local quantum conformal theory reveals itself to be ghost-free, so the unitarity of the theory should be safe. After the construction of UV-finite theory, we focused on making it manifestly conformally invariant using the dilaton trick. We also argue that in this class of theories conformal anomaly can be taken to vanish by fine-tuning the couplings. As applications of this theory, the constraints of the conformal symmetry on the form of the effective action and on the scattering amplitudes are shown. We also remark about the preservation of the unitarity bound for scattering. Finally, the old model of conformal supergravity by Fradkin and Tseytlin is briefly presented.
\end{abstract}

Keywords: quantum gravity; conformal gravity; quantum field theory; non-local gravity; super- renormalizable gravity; UV-finite gravity; conformal anomaly; scattering amplitudes; conformal symmetry; conformal supergravity

\section{Introduction}

From the beginning of research on theories enjoying invariance under local spacetime-dependent transformations, conformal symmetry played a pivotal role-first introduced by Weyl related changes of meters to measure distances (and also due to relativity changes of periods of clocks to measure time intervals). Weyl thought of transformations changing the scale and quite boldly he considered them not only in global version (where the parameters of the transformations are constant), but also in local (where the parameters depend both on space location and time). Then, he also understood that just rescaling transformations

$$
d s \rightarrow d s^{\prime}=e^{\lambda} d s
$$

(which form abelian group) after making them local gives rise to full conformal group of transformations, where only angles remain invariant, but the sizes, magnitudes and scalar products between vectors change. These primordial considerations were done within a set of ideas that conformal transformations are the symmetries of fully relativistic consistent classical theory of gravitation. However, Einstein beat the Weyl theory with his famous second argument about radiating atoms in various gravitational fields. With the triumph of Einsteinian relativistic theory of gravitation (confirmed by the measurements done during the solar eclipse in 1917), which includes only symmetries with respect to diffeomorphism transformations, Weyl's conformal theory of gravitation was relegated and treated as non-physical. However, the ideas of scale transformations seeded by Weyl were revealed to be very fruitful in the development of local gauge theories. The change was that 
the transformations were done in the internal space, not in spacetime, and that they were imaginary rescalings or simply complex phase transformations compared to the ones in Equation (1). This with London equations started a revolution leading to $U(1)$ gauge theory known as electrodynamics and even to non-abelian generalizations as embodied by Yang-Mills (YM) theories. However, the origin of the German word "gauge" used now so often in field theory is clear and undoubtedly points to different real scales used for measurement of distances, like for example between two rails in rail transport.

It seemed that in the classical world conformal symmetry had to play a rather small role and was not used in relation to classical gravity. The time for conformal methods in general relativity (GR) had to come later, for example when the research on black holes and their spacetime causal structure culminated in the golden age of classical gravitation in the 1960s and 1970s. In a different vein, conformal invariance and conformal symmetry started to become appreciated a lot also in the era of modern quantum field theories. With the development of the renormalization methods in perturbative quantum field theory models, conformal symmetry became an indispensable tool for every particle physicist. Moreover, the relation between Renormalization Group (RG) flow and Conformal Field Theories (CFT) and conformal anomalies was found to be even more tight due to the understanding of physics at and near a fixed point of RG. The application of conformal considerations in field theory was also beautifully unified with gravitational (spacetime) considerations through AdS/CFT duality. Now, every modern theoretician knows that conformal tools are both useful and enlightening for investigations both in gravity and in field theory. In the advent of bigger understanding of conformal symmetry, we could come back and refresh interest in the original Weyl gravity, where the original conformal symmetry is taken as a basis for the theory of gravitational interactions. However, on the quantum level, we meet several serious obstacles. Actually, one can find that there is a very special relation between quantum physics and conformal symmetry, which is not enjoyed by any other known symmetries of Nature, nor by other gauge symmetries used in Nature.

To get effective field theories (EFT), we typically need to perform a very abstract mathematical procedure called quantization. It takes a classical action of a theory and enforces the rules of quantum mechanics. In all perturbative approaches, the result of this artificial procedure done only by theoreticians (because the Nature is already always quantum!) is expressed as a perturbative series in some small parameter. Typically, this is a Planck constant $\hbar$ multiplying some couplings of the theory and this is equivalent to the loop expansion. It is necessary here to distinguish the two possible meanings of EFT: they appear either as a low-energy effective description (in powers of a small length scale) or as a quantum effective description (in powers of Planck constant). Now, the question arises of how to find effective actions of a given model at any loop order. By the knowledge and experience gained in some ordinary QFT, this is a very complicated process and the effective actions at higher loops are non-local functionals of fields, and of covariant differential operators and typically with infinite number of terms hence almost impossible to handle exactly without employing any approximations. One may think that the gauge symmetries of the theory (if they are non-anomalous on the quantum level) may help in constraining the number of terms in the effective action. However, since in EFT, we do not have to obey the renormalizability conditions at loop orders, the number of terms is still infinite, and all of them can be written in a gauge covariant fashion. In the act of resignation, we may contemplate the details of the procedure of quantization transforming some classical action functionals into much more complicated non-local functionals at one-loop, two-loop and higher loop levels. Someone may think about taking some shortcuts and for example considering two-loop effective action as a result of quantization applied to one-loop level of already quantized one-loop level effective action. This is not fully correct and such one-loop RG-improved actions are valid only approximately. However, one shall focus on the possibility of an existence of some fixed point action functional of such procedure of obtaining higher loop corrections.

In addition, here is the place, where the conformal symmetry enters for the first time in QFT. It happens that, when the conformal symmetry is present and non-anomalous on the quantum level, 
then the above procedure is at the fixed point, or, in more mathematical words, the quantization procedure is here idempotent and the full effective action (with all perturbative orders resummed and also with non-perturbative contributions) is here identical to the classical action of the theory that we started with. This is a very luxurious situation, when the quantum theory looks exactly the same as the classical one. Such examples are difficult to find, but one is already known for a long time. This is an $\mathcal{N}=4$ super-Yang-Mills theory (SYM), which is very symmetric and moreover conformal on both classical and quantum level in $d=4$ spacetime dimensions. The other models result as deformations to the gauge structure of $\mathcal{N}=4$ SYM theory and therefore this theory can be viewed as a "harmonic oscillator" of the theoretical physics in the 21st century. All other theories may look more realistic, but their theoretical description and understanding that we have about them is only a direct consequence of what we understand about this conformal theory and a simple afterward application of a conformal perturbation theory to some operators which deform the original structure. The explanation why conformal symmetry is essential here for having very nice form of the effective can be found below.

There is possible a question why theories, which are conformally invariant on the classical level, cease to be such on the quantum level. One may blame the fact that the quantization procedure of classical field theories does not preserve conformal symmetry, but the situation can be in general more complicated. If the symmetry is not present on the quantum level, but it was present on the classical one, then we say that the theory is anomalous. There could be three distinct reasons why the absence of some symmetry may appear at quantum level:

1. First, the obvious reason is if the classical Lagrangian of a field theory model does not possess the original symmetry and this is also inherited by quantum dynamics.

2. The second reason is related to that, in the definition of quantum theory via path integral, we have to integrate over field space with some specific measure. In addition, this measure may lead to anomalies as explained in the Fujikawa method of deriving, for example, a chiral anomaly.

3. The last reason is that, in the process of defining the quantum theories and absorbing ubiquitous UV-divergences, we need to specify a renormalization procedure to give physical meaning to all infinite results of the theory. This procedure also leads to anomalies, if the scheme of renormalization is not chosen adequately for the symmetries present in the classical theory.

In the last two cases, one says that the quantum anomaly shows up. Generally speaking, the presence of the quantum anomaly may be disastrous for the definition of quantum theory, if, for example, in the proof of renormalizability, this symmetry is used via Ward-Takahashi or Slavnov-Taylor identities. Therefore, gauge or gravitational (and also mixed gauge-gravitational) anomalies should be all cancelled in consistent models of QFT. This is done by careful examining the spectrum of all particles of the theory, their quantum numbers and the structure of their interactions. In addition, for example, the standard model of particle physics (SM) coupled to Einsteinian gravity is anomaly-free theory but not all of its extensions are. Finally, the requirement for anomaly cancellation is a strong condition for model building. Furthermore, we notice that the absence of anomalies is a condition pertaining to loop orders in expansion of quantum dynamics and, at tree-level, such requirement does not seem essential. This means that we can freely and correctly construct all tree-level $n$-point functions for the theory (which correspond all to classical level) without bothering about the problems of invariance of the measure in path integral or a renormalization scheme which could preserve all symmetries. There the output is simple and classical: if the theory is with symmetry classically, then all $n$-point Green functions will enjoy this symmetry and there is no issue of anomaly. On the contrary, on the quantum level, the anomaly may show up (and does indeed in anomalous theories) and this can be seen for example by evaluating famous one-loop triangle diagrams.

What about conformal anomaly? How does this show up in the models of QFT? The short answer is that it shows in UV-divergences of the theory [1-4]. They are quite common in QFTs so the immediate conclusion is that many QFTs are with conformal anomalies. However, most of the time, this is not crucial for features of quantum theories since physicists got used to dealing with infinities via 
a renormalization programme. In addition, since typically for matter (e.g., non-gravitational) theories on the classical level, conformal symmetry was not gauged (was not made fully local), then this was not a gauge symmetry of these models and was not essential on the quantum level in proving Ward identities, for example. For matter theories, conformal invariance was some feature of the classical theory generically not enjoyed any more by the quantum dynamics, which is in turn based on quantum non-anomalous matter gauge symmetries. The notable exception here is mentioned above $\mathcal{N}=4 \mathrm{SYM}$ theory, which is not only quite highly supersymmetric (eight generators of supersymmetry) but also conformal on both classical and quantum level. However, here in such superconformal theories on flat Minkowski spacetime background, the conformal symmetry is not gauged.

It is quite easy and intuitive to understand why conformal symmetry has to do with divergences of the theory, when the upper UV limit of loop integrations is taken to infinity. First, we noted that, in a very popular dimensional regularization scheme (DR) of renormalization, it is customary to change the dimensionality of spacetime by a small amount. However, conformal invariance of the theory is very sensible to such even small manipulations and a theory which was originally on the classical level conformally invariant in $d=4$ spacetime dimensions behaves as not conformally invariant in other dimensions (typically in $d=4-\varepsilon$ ). This is related to particular constraints that the conformal invariance puts on the terms in the Lagrangian and we write more about this also below. Simply, if we do not have divergences, then in DR scheme, we do not have to leave $d=4$ dimensions and the theory if conformal remains such also on the quantum level. Another advantage of using DR is that some infamous UV-divergences which are not scheme-independent (we mean power-law divergences) do not beset our renormalization programme in this approach and we have to deal, and renormalize, and absorb only logarithmic divergences in the cut-off $\Lambda$ (put as the upper limit of integrations over loop momenta).

There exists also another argument which links conformal invariance and the absence of UV-divergences (which is also called UV-finiteness of the theory). If the theory is truly scale-invariant (which is the first step towards conformal invariance), then the upper limit of loop integrals can be freely rescaled so the result of the integration is independent on $\Lambda$. If it is really independent, then it means that it is not divergent because the actual value of the integral can be taken in the case, when the regulator $\Lambda$ is still finite. In such conditions, there are no divergent parts of the integrals and only finite parts are computed in the way described above. The theory is finite, without divergences, all the beta functions vanish and the conformal symmetry is present on the quantum level, if the classical Lagrangian of the theory was conformally invariant too.

Another point of view is to relate divergent parts of the Green functions computed at loop levels to the divergence of the conformal current. This gauge current $j_{\mu}$ describes how the theory changes when the scales of momenta of all particles of the theory are rescaled. If the theory possesses a conformal anomaly on the quantum level, then this current is not conserved in the operatorial sense in Hilbert space and even in the sense of quantum expectation values. This has also to do with the trace of the effective energy-momentum tensor of matter fields considered as a quantum operator. If this tensor with quantum corrections is trace-free (in the sense of quantum vacuum expectation values of the corresponding operator), then we have conformal symmetry present on the quantum level as well.

The last thing that we would like to discuss here is the issue of conformally invariant regularization of some QFT models. As emphasized above, if the theory produces UV-divergences at the quantum level, then it cannot be conformally invariant. However, there do exist conformally invariant regularizations and conformally invariant forms of the divergent parts of the effective actions. (They can be obtained by applying a trick with conformal compensator to any generally covariant terms in the action). However, as mentioned above, it is only one of three needed conditions that is satisfied here. The three conditions are needed in order to have full conformal invariance on the quantum level. The other two were the conformal invariance of the classical action and of the field measure in the path integral. Only all three conditions together secure the conformal symmetry on the quantum level. If the conformally invariant regularization is used and even if the divergent parts of the effective action are written in the conformal 
way, still the theory is not conformal on the quantum level because still it contains UV-divergences. This basically means that the integration measure of the path integral in such models is not preserved by the conformal transformations. We can give here a simple example of one-loop situation in the ordinary four-dimensional YM theory. The divergences are there and they can be nicely collected into a divergent part of the effective action taking the gauge-invariant form

$$
\Gamma_{\mathrm{div}}=\frac{c}{\varepsilon} \int d^{4} x \operatorname{tr} F^{2},
$$

where $c$ is some non-zero constant coefficient depending on the precise structure of the gauge group and $\varepsilon$ is the regulator in DR. Actually, here we notice that, due to the renormalizability of YM theories in $d=4$ spacetime dimensions, this action takes the same structural form as the classical action of YM theory. We remind readers that, from the time of Bateman and Cunningham, it is known that in $d=4$ (and only there) the $F^{2}$ action is classically conformally invariant. This endorses that also our procedure of renormalization of one-loop quantum divergences is conformally invariant, since the action $\Gamma_{\text {div }}$ is. (Here, we do not even need a conformal compensator to achieve this). However, everyone agrees that pure YM theory is not conformal on the quantum level and this can be traced back to the fact that the divergences are indeed present, even if written in a conformally invariant manner! The appearance of UV-divergences (regularized in whatever way, even in the conformal one) shows that conformality is broken and this is another side of the same coin. If we carefully look at the other side, we see problems with the conformal invariance of the measure in the path integral. The absence of UV-divergences or vanishing of the beta functions (or in the language of the RG community the condition of being at a fixed point of RG) are all signals that we are dealing with conformally invariant theories on the quantum level. These all are different sides of the same coin that can be investigated also from the technical point of view by the analysis of invariance of the measure, of the quantum renormalization procedure and of the classical action of a theory.

The first prerequisite for conformal theory on the quantum level is to have a classically conformally invariant theory. In addition, for this, the very simple requirement is classical scale-invariance. This means in turn that, in the classical Lagrangian of the theory, we cannot have any dimensionful parameters, like masses of fields or dimensionful coupling constants (like the Fermi coupling of weak interactions). If in the theory we have one mass scale, then this is equivalent to having many of them because we can always rescale it to get any other energy scale at our wish. We will also comment later that classical scale invariance is quite restrictive and can determine the form of the action of a theory to some big extent. For example, the requirement of the absence of dimensionful couplings in any dimension $d$ first gives rise to renormalizable models. However, the situation with conformal symmetry on the classical level puts even more constraints and the renormalizability on the quantum level may not be achieved. Classical scale-invariance of the theory requires no mass for fields or any other scales in parameters needed for the definition of the theory. One may think that the same should roughly happen on the quantum level, where in the definition of the theory we may have addition of new scales (related to renormalization $\mu$ ). The whole point about quantum conformal theories is to avoid the introduction of this new scale $\mu$ and then we have exactly the same situation as on the classical tree-level-no mass parameters in the definition of the theory. This situation with enhancement of symmetries on the quantum level is the reason for miracles and simplicity of quantum CFT.

After discussing at length the issues related to the conformal anomaly and its effects on the quantum dynamics, now we come back to the situation with conformal symmetry in quantum gravity. As any symmetry in quantum gravity, this invariance has to be gauged on the quantum level $[5,6]$. If someone tries to marry conformal symmetry in gravitation with quantum mechanics, then one encounters very serious problems with consistency of a such framework. Not only merging of relativistic gravitation with quantum field theory is difficult, but on top of problems there, we also 
have to make the full theory consistent with local conformal symmetry (invariance with respect to Weyl rescalings of the metric tensor:

$$
g_{\mu \nu} \rightarrow g_{\mu \nu}^{\prime}=\Omega^{2} g_{\mu \nu},
$$

where $\Omega$ is an arbitrary function of spacetime points $\Omega=\Omega(x)$ ). In the language of anomalies, we must ensure that, in a consistent quantum theory, all gravitational and conformal anomalies are precisely cancelled. This regarding the conformal part of the full symmetry group (which will consist here of diffeomorphism and local conformal transformations) means that, in such theory, on the quantum level, there are no UV-divergences at any loop order. This is a very strong constraint, almost impossible to be achieved in models of quantum gravity often considered in the literature. It is desirable to find a theory, in which all beta functions (perturbative as well as non-perturbative contributions) are exactly zero and such theory can be viewed as a gravitational gauged analogue of the famous and very well behaved on the quantum level $\mathcal{N}=4$ SYM theory. The fact that all beta functions are tuned to zero is an expression of very high fine-tuning but also all superconformal theories are highly fine-tuned. In the quest for quantum conformal gravity theory, the consistency requirement of the absence of conformal anomaly is very crucial since this symmetry is gauged. Moreover, it constrains quite a lot of possible searches for candidate conformal quantum gravity. This local symmetry is instrumental in proving Ward identities (here conformal Ward identities) and they are very important elements in the description of a quantum dynamics of such a system. If we do not have conformal anomaly of precisely zero, then all features and advantages of conformal symmetry on the quantum level are inaccessible and lost.

We need conformal invariance preserved at the quantum level of the theory because this is the level which decides about very important features of the theory, its renormalizability, control of the divergences and unitarity. As it is known for particle physics, it is desirable to have very nice properties of the theory per se, while the specific choice of the vacuum, asymptotic states or background solutions of the theory may not respect all of them and may lead to breaking of some symmetries of the theory. However, for theoretical considerations about the situation in a theory, it is better to have as many symmetries as possible since the symmetries constrain the dynamics, lead to integrability or even to solvability of some models.

In the search for a conformal quantum gravity, we may take various attitudes and various directions. First, we may desire to analyze the situation with standard Einsteinian gravity as described by Einstein-Hilbert action in $d=4$ spacetime dimensions:

$$
S_{\mathrm{E}-\mathrm{H}}=\int d^{4} x \sqrt{|g|} \kappa_{4} R .
$$

However, such theory (with or without a cosmological constant term which could be possibly added here) reveals itself to be non-renormalizable so the problems here with UV-divergences are even more severe. We write a detailed account on UV-divergences and their history in this theory in the section about infinities. Although it is known that in $d=4$ spacetime dimensions this theory on the classical level is not scale-invariant, because of the presence of a dimensionful coupling constant $\kappa_{4}$ related to the Newton's constant, the situation can be improved a bit by using a trick with a dilaton field. Such dilatonic Einsteinian gravitation is indeed scale-invariant classically, but the UV-divergences are inevitable on the quantum level and they destroy the conformal symmetry. We consider more about the dilaton trick also in Section 3.

This review is organized in the following way. In the next section, we review the long story of infinities which at the end led to the first construction of UV-finite gravitational theory. Then, we comment on the conformal quantum gravity, its situation at the one-loop level and also its scattering amplitudes (both at the quantum and classical levels). Finally, in tight relation to previous topics, we discuss some virtues of the Fradkin-Tseytlin conformal gravity models in four dimensions. 


\section{Long Story of Infinities}

Interacting QFTs were always plagued by divergences. They appear both in the UV as well as in the IR limit of integration over loop momenta. Here, we will consider only infinities showing up in the UV limit of the theory. They are present in almost any model of QFT, with the notable exceptions of UV-finite theories, which are convergent in the UV regime. Original hope was that, in fully consistent QFT, we will be able to avoid all these divergences and the theory will give us only finite convergent results. However, soon after the development of first interacting models of QFT, such an idea was abandoned as too naive. The problem of infinities motivated and strongly shaped the research in particle physics and field theory in the last 70 years. They led to introduction of new research directions of renormalization and renormalization group flows. The understanding of them forced us to change our views on the parameters of the physical theory and on how to distinguish between bare (theoretical and unobservable) parameters and effective and measurable (physical) parameters. Moreover, new tools had to be introduced to describe quantum physics, like regularization methods and beta functions. Actually, a particular area of research in theoretical physics evolved into a whole new field of quantum RG flows. These developments also gave a little help to our big quest of classification of quantum field theories and, thanks to various problems with UV-divergences, we were able to distinguish two classes of theories: renormalizable and non-renormalizable as first discussed by Pauli. Since the first distinction was based on simple analysis of the energy dimension of couplings, the further progress led to refined notions of renormalizability and also non-renormalizability.

Before we get to the UV-finite gravitational theories (so theories completely without divergences), let us first briefly review the situation with divergences in other theories of gravitational interactions on the quantum level. These theories were arising due to historical reasons in the following order. First, gravitational theory based on Einstein-Hilbert $(\mathrm{E}-\mathrm{H})$ action

$$
S_{\mathrm{E}-\mathrm{H}}=\int d^{d} x \sqrt{|g|} R
$$

was quantized and the situation with divergences was analyzed. Since the conclusions were very pessimistic, this motivated many researchers to change and modify the classical theory and start with a quantization of some modified gravity models. The big promise arouse with four-derivative theories in four-dimensional spacetimes since they were the first models to be proven to be power-counting and multiplicatively renormalizable. Indeed, the modifications of gravity, which includes terms with higher number of derivatives revealed to be fruitful in gaining control over infinities at the UV limit. For example, gravitational theories with ten derivatives in four spacetime dimensions revealed to be one-loop super-renormalizable meaning that the control over divergences was highly strengthened. In such models, the divergences appear only at the one-loop level, while at higher loops the results of all integrals are convergent. Theories with higher derivatives (HD theories in short) are typically beset by the problem of ghosts and to solve this issue non-locality was invoked. In non-local theories, the problem of ghosts does not show up, but at the same time good control over divergences can be still kept. Actually, it is possible to construct such non-local gravitational theories for which the behaviour of the theory in the UV limit coincides exactly with the behaviour of one-loop super-renormalizable theories. Then, the final step in the construction of UV-finite theories is to add some operators called "killers" to "kill" completely the remaining beta functions at one-loop level. Actually, we will see that, in UV-finite theories, the non-locality is not a must and the theory can solve all the problems of higher derivative formulation, if it is conformal on the quantum level. However, this is the chronological path and we will follow such in the presentation here.

First, we consider UV-divergences in E-H theory in $d=4$ four dimensions. This theory can be quantized in a standard way using the Faddeev-Popov methods on the path integral. The result of such covariant quantization is a QFT with one coupling, which is a mass parameter $\left(M_{\mathrm{Pl}}\right)$, which is the square root of the inverse of the gravitational Newton's constant $G_{N}$ (up to some numerical coefficients). As such, it is a dimensionful parameter in the theory, which may signal a forthcoming 
problem with infinities and renormalizability. This is verified and confirmed by the careful analysis of perturbation calculus in such a theory, which is actually a series in $\kappa_{4}=M_{\mathrm{Pl}}^{-1}$, which in turn has the inverse mass dimension. According to the criterion by Pauli, such theory is non-renormalizable (at least on the perturbative level) since this series reminds a series one gets if perturbatively study Fermi's theory of weak interactions. The E-H quantum gravity has serious problems with divergences-at least this is what the naive power-counting analysis is telling us. However, maybe we should be more careful and do not always trust the worst behaviour given by the power-counting.

Indeed, in 1974, 't Hooft and Veltman were more careful and with a big effort [7] computed explicitly the form of divergences at one-loop in quantum gravity. Their divergent part of the off-shell effective action takes the form:

$$
\Gamma_{\text {div }}^{(1)}=\frac{1}{8 \pi^{2}(4-d)} \int d^{4} x \sqrt{|g|}\left(\frac{1}{120} R^{2}+\frac{7}{20} R_{\mu \nu}^{2}\right) .
$$

Their setup was the following. Their theory was quantum Einstein-Hilbert without matter (pure gravity) treated perturbatively at one-loop in four dimensions. They also analyzed the situation for both on-shell and off-shell Green functions of the theory. What they found is that, contrary to the expectations expressed above, such model was completely convergent for everything that regarded the on-shell (using classical vacuum equations of motion of the theory) $n$-point functions. This is truly a "miracle" since, at one-loop, this theory is UV-finite. (It is not merely a non-renormalizable one, it is one-loop finite!) Thus, 't Hooft and Veltman achieved finiteness of E-H theory already in 1974. This is not true because the "miracle" does not always happen, especially if we want to move in any direction away from the setup considered by these authors. Moreover, the reasons (mathematical and physical) can be understood for the "miracle" to happen and a deeper analysis shows that, in a more general situation, we should not expect anything like this to happen again.

Now, thanks to 't Hooft and Veltman, we can explain the result with a complete absence of divergences at one loop. This is due to two facts. The first is the possibility of performing gravitational field redefinitions for on-shell Green functions on the backgrounds, which are gravitational vacua (that is are Ricci-flat in E-H theory not coupled to any matter). The second fact is that, in $d=4$, we are lucky and a variation of a celebrated Gauss-Bonnet term

$$
\mathrm{GB}=\text { Riem }^{2}-4 \mathrm{Ric}^{2}+R^{2}
$$

is a total derivative, and hence can be neglected under spacetime volume integral (if the proper boundary conditions are used or the fields of the theory fall off sufficiently fast at asymptotic infinity). We use the GB density in the action integral of the theory at the one-loop effective level and, by variation of the last object, we derive equations of motion (EOM) and two- and higher $n$-point Green functions. For such purposes, if something is a total derivative, then it can be safely ignored. This last fact has to do really with the number of dimensions because another way of writing the Gauss-Bonnet term is

$$
\mathrm{GB}=\frac{1}{4} \epsilon_{\mu \nu \rho \sigma} \epsilon_{\kappa \lambda \tau \omega} R^{\mu \nu \kappa \lambda} R^{\rho \sigma \tau \omega}
$$

and there we explicitly see the use of the completely anti-symmetric Levi-Civita tensor $\epsilon$. Such tensor with four indices can only be defined in four dimensions and in other dimensions we have a mismatch between the number of indices on two Riemann tensors (eight indices) and on two epsilon tensors (in general case $2 d$ indices). This explains why four dimensions are special regarding the properties of the Gauss-Bonnet term. Altogether, these two facts make the on-shell part of the divergent action at one-loop level in pure E-H quantum gravity vanishing, so there is no a problem of divergences there.

However, the "miracle" is gone, if we try to consider more general setup. If we change the number of dimensions to $d \neq 4$ (typically to $d>4$ ), then in such new dimensions there is nothing like GB identity and there is no reason to expect absence of divergences. If we couple matter to pure E-H gravity, then Ricci-flat manifolds are not background exact solutions of the theory and we cannot use 
vacuum field redefinitions any more. Similarly, if we go off-shell and study amplitudes for virtual external particles, again we cannot redefine the metric field there. Finally, the situation needs to be investigated at the two-loop order. First, naive power-counting analyses suggested that we should expect divergences with energy dimension 6, so a corresponding term in the divergent part of the effective actions would contain six derivatives on the metric tensor.

Algebraic considerations by Nieuwenhuizen [8] and others showed that, in vacuum (Ricci-flat) solutions, all algebraic tensors that can be constructed with six derivatives reduce to tensors, which are cubic in gravitational curvatures and moreover, due to assumed Ricci-flatness, they all can be written as cubes of the Riemann tensor (various contractions). Actually, the usage of some dimensionally dependent identities reduces this algebraic space to only one element, which is conveniently represented using a traceless Weyl tensor. (Since we get the Weyl tensor from Riemann by subtracting some Ricci tensors or scalars, this change on Ricci-flat backgrounds amounts to nothing). This one scalar cubic invariant in Weyl tensor we denote schematically by $C^{3}$ and its explicit form

$$
C^{3}=C_{\mu \nu}{ }^{\rho \sigma} C_{\rho \sigma}{ }^{\kappa \lambda} C_{\kappa \lambda}{ }^{\mu \nu}
$$

shows some kind of cyclicity and symmetry of it. (This invariant looks like a trace of Weyl cube tensor treated as a matrix in its pairs of indices. Moreover, the contractions of indices occur exclusively parallely in pairs). Hence, the question was whether such term could appear in the on-shell part of the divergent effective action at the two-loop order in pure quantum gravitation based on $\mathrm{E}-\mathrm{H}$ action. By a very tedious computation, Goroff and Sagnotti in 1985 showed that the coefficient of the counterterm needed to absorb divergences appearing at this level is non-vanishing [9-11]. We just quote the exact figure for the reference here (but important is only the fact that it is non-zero):

$$
\Gamma_{\text {2-loop, on-shell }}=\frac{209}{2880(4 \pi)^{4}} \frac{1}{4-d} \int d^{4} x \sqrt{|g|} C^{3},
$$

where this result was obtained in a dimensional regularization (that is why the factor $d-4$ appears above). This shows that also at two-loop level the "miracle" is gone. This is to be expected since we do not have any strong reason to exclude UV-divergences of this form at this level. All facts about field redefinition and speciality of four dimensions cannot help in reducing algebraically the invariant $C^{3}$ and, since it is algebraically possible, then it will be generated by quantum corrections. (This is some kind of a Murphy's law of QFTs: if some divergent term is not excluded by the symmetry or algebraic considerations, then sooner or later it will appear in quantum computation).

In the renormalization of the E-H theory at the two-loop order, we have to be prepared to absorb any divergence, which appears due to quantum corrections and this divergence requires quite exotic covariant counterterm (with six derivatives) - compare this one with the original E-H action containing two derivatives. The non-zero coefficient above signifies that the pure E-H theory is non-renormalizable at the two-loop order and the naive expectations from power-counting analysis hold true. To renormalize such theory, even on-shell, we need a counterterm of the covariant form $C^{3}$ and such was not present in the original action.

We also note that, from the two-loop level, the expectations based on schematic power-counting analysis are true and the theory behaves quite badly regarding the control of UV-infinities. When we increase the loop level, the terms arising as divergences have higher powers of energies, so we need more derivatives to absorb them in covariant counterterms. The control over divergences is here completely lost and the original hope for a consistent QFT of gravity drifts very far away. We cannot take $\mathrm{E}-\mathrm{H}$ action as a basis for quantum field theory of gravitational interactions. This theory behaves very badly on the loop levels, although it can do well as an effective theory (in the first approximation). Besides the very theoretical problems of non-renormalizability, the real physical problem is mentioned above non-predictiveness and this has to do a lot with experiments. Actually, a careful reader can 
notice that, due to the renormalization, such theory on higher loop levels makes the same predictions as a sufficiently complicated theory with higher derivatives.

The situation with divergences is very bad in the initial gravitational theory. What could help us to overcome these problems? First, a bit conservative solution is to add more symmetries to the theory. This is fruitful, when symmetries joining fermions and bosons are added because they are known from playing a crucial role in cancelling divergences as a result of fine-tuning of interactions between different fields, but now residing in the same symmetry multiplets. For the case of gravitation, the addition of supersymmetry (here in local version) leads to supergravitation theories. These theories on the quantum level indeed behave better than their just pure gravity counterparts. The situation is improved a little due to gravitinos and their gravitational interactions. Since they are fermions, loops with them contribute with minus signs and this opposite sign reveals to be important for cancellation of some divergences. However, not all divergences are cancelled. What is worse: not all divergences are even under control. In original supergravity models with two or four local supersymmetries, we know that, at a sufficiently high number of loops, the divergences show up and they are not possible to be absorbed in the original two-derivative action, due to the arguments pretty much the same like elucidated above. Hence, the theory reveals to be non-renormalizable, not only non-finite, so original hopes are gone.

In turn, the situation in supergravities with high number of supercharges, like in $\mathcal{N}=8$ in $d=4$ is still not settled down, due to the very complicacy of the theory and it is not known, if the theory is with divergences at five loops, for example [12-15]. The quite high level of loops is, of course, another tremendous difficulty here. Original and old conjectures were that such theory may reveal to be UV-finite; however, if the divergences are present, then it will be non-renormalizable like any other (super-) gravity theory in $d=4$ based on two-derivative action in pure graviton sector. Indeed, this is true, that all supergravities theories discussed so far in this paper in the gravitons sector coincide with a theory based on E-H action, since they all have two derivatives. The logic as laid down above will apply to them too, since in their actions they have two derivatives only, and in four dimensions this is too little. We think that right now it is likely that even very highly constrained by local supersymmetries (and other hidden symmetries) models of supergravity with two-derivative actions will show at sufficiently high level of loops divergences, which cannot be absorbed in the existing terms of two-derivative theory. Actually, the problems there are formulated in a form of questions, whether the counterterms can exist or not at all, due to the all existing and constraining symmetries. The miracles at one- and two-loop levels are due to the facts that some counterterms (like $C^{3}$ ) in supergravity cannot be written in a (super-)symmetric way, so they cannot show up and in the result there are no divergences coming with them. However, the superspace formulation does not exist for theories with $\mathcal{N}>1$ supersymmetries, so it is also very difficult to use such covariant formal arguments. We leave the path of highly constrained two-derivative supergravity theories, since now they do not offer very convincing and satisfactory answer for the problems of UV-divergences.

Another option is to try to employ conformal symmetry already on the classical level in gravitational theory. However, since E-H is a theory with only diffeomorphism symmetry which is gauged, this is difficult. Some attempts can be done with a dilaton field (treated like a dynamical conformal compensator), but generally the situation with infinities is not improved. In addition, this can be traced back to the fact that the gravitational coupling constant has dimension of mass or equivalently the dilaton field takes a vacuum expectation value (v.e.v.). If we have a theory with a mass parameter, it cannot be on the classical level scale-invariant. Strictly speaking, here we have a vacuum state of the theory, which is not scale-invariant, but the full theory is scale-invariant. Hence, we do not start with a setup for a conformal gravity theory on the classical level. If we manage to have such a theory as the starting point, then another pending question is whether it remains conformal also on the quantum level. This question is even more important than the original one, since we have examples of classically conformally invariant theories, which are not any more scale- and conformally invariant on the quantum level, like the standard two-derivative YM theories in four dimensions. 
There could be also a possibility that a theory, which is not classically conformally invariant, becomes such after inclusion of all quantum corrections, but, until recently, we have not known any example of such a behaviour. Such quantum enhancement of symmetries actually appears in the quantum gravitational setup that we are going to consider in this paper. The examples of such enhancement of symmetries on the quantum level are already known in the theory of critical phenomena. There, due to quantum RG effects, the special point at sufficiently high energies is met. The symmetry of the theory at this conformal fixed point of RG flow is bigger than the original classical theory at small energies. The quantum effective action at this critical point can be viewed as a resummation of perturbative quantum contributions with all effects of RG taken into account. This effect can be clearly seen as the non-perturbative one (or as we want to phrase it as arising from resummation). The situation with the theories of quantum gravity that we analyze in this review is such that already at the one-loop level all contributions of perturbative loops are summed up (at least regarding UV-divergences). This amazing feature is due to the super-renormalizability property of higher derivatives theories that we are concerned with in this paper. The quantum enhancement of symmetries could be also realized, if the dilaton field dynamically decouples and drops out from the effective action of the theory. In a sense, this would be the reverse of a dimensional transmutation process, which in some theories without scales produces new scale, which arises dynamically, like the famous $\Lambda_{\mathrm{QCD}}$ scale arising in the theory of strong interactions.

It is clear from the preceding discussion that we must modify the gravitational theory on the quantum level, and by this we do not mean adding more symmetries, but change completely the underlying dynamical principles governing quantum gravitational field. Below, we stipulate conditions that we want to preserve, when putting modifications to gravitational theory on the quantum level. First, we want to use and accept standard rules of Quantum Mechanics (QM) and, as a consequence, we will modify gravity part, keeping the quantum mechanical part untouched. Following these rules, we want to cast the quantum theory of the gravitational field in a framework of very successful (so far) QFT. To quantize a classical system with gauge (here diffeomorphism) symmetries, we will use standard covariant rules of quantization of gauge theories. We can use, for example, a path integral approach to quantize the theory and to obtain generating functionals for Green functions. For dealing with gauge symmetries, we will use a standard Faddeev-Popov trick of quantization of gauge symmetries. In this way, we hope to get a standard version of QFT of gravitational interactions. As marked above, we will use covariant treatment, which manifestly preserves Lorentz symmetries and, moreover, is background-independent-this is why we will use a formalism of background field theory. Additionally, we want to exploit the power of perturbative framework of QFTs. At the end, what we want to get is a consistent QFT framework for gravitational interactions.

From the point of view of particle physics, we propose a QFT, whose particle excitations correspond to structureless and point-like massless gravitons with two helicities and with the spin 2 . Setting the rules above, now we take the step to modify classical gravitational theory, which is later quantized according to the stipulated rules. We modify the gravitational theory by changing its action, since the action defines completely the dynamics for field theory systems. We decide to take this step as an act of desperation, since all other attempts failed to give us satisfactory answers for the problem of UV-divergences in quantum gravity theory. We know that, in the EFT framework, Einsteinian gravity is a good physical theory, but we are also aware of its limited range of applicability. It works very well on the classical macroscopic level (like the distances within our solar system). However, it does not describe the dynamics very well at the very small scales (microworld) and at very large scale (cosmology). Therefore, it is not surprising that we must modify this theory at those scales and that a more general theory should be used for quantization programme, while the Einsteinian gravity should be recovered only in the classical macroscopic limit and for low energies.

We desire the following modifications done to the gravitational theory. We modify the gravitational action of a theory in order to satisfy many different requirements (some of them really sound like perfect wishes). Namely, we want a self-consistent theory of massless spin-2 interactions 
on the quantum level, so the theory must be written using covariant language, using gravitational curvatures and covariant derivatives only. Such theory should be defined at any energy scale, so we should not find any problems, neither in UV, nor in the IR limit of the theory. This theory should be possible to be formulated in four-dimensional spacetime. This does not preclude that we will not accept an idea of extra dimension(s). We just want to say that the theory should be first defined in four dimensions with the possibility for extension, it should not be only constrained to $d=4$.

As for the construction of a gravitational theory, we want to use only metric degrees of freedom and we will not allow for dynamical torsion or non-metricity. However, in the gravitational spectrum of the theory, we may want to accept spin- 0 and spin- 1 particles as well, together with a dominant massless spin-2 contribution from gravitons. Regarding the strictly quantum properties, we want a theory written in a QFT language, which will lead to unitary $S$-matrix for scattering processes between on-shell asymptotic states of the theory. Since Lorentz-invariance is well tested experimentally and holds true to very high energies, we also want to preserve this symmetry of Nature in our construction. Lorentz or more generally Poincaré symmetry is a symmetry of asymptotically flat spacetimes, hence this symmetry pertains to the definition of asymptotic states, which are used for the construction of the S-matrix. We do not want to break this good and useful symmetry either. Moreover, in the same spirit, but on general backgrounds, we do not want to single out any particular geometrical structure, hence in the middle of our manifold (in not asymptotic regions) we have to be able to write our theory in a generally covariant and background-independent fashion. As motivated extensively above, we must write a new gravitational theory using terms with higher derivatives added to the E-H action. We will also soon give another arguments, why higher derivatives are inevitable in quantum gravitational theory. In addition, finally we express our desire that the situation of this theory in the UV is much more improved compared to the original E-H theory. We want to build a theory, which is UV-complete in the high energy regime, where the full control over UV-divergences is gained and which is renormalizable, super-renormalizable, and finally the most difficult wish is to require from such theory to be UV-finite. In the next pages, we will show that it is indeed possible to increase the control of infinities and eliminate them completely in a sequence of steps as mentioned above.

The final argument, which will be used for the inclusion of terms with higher derivatives in the gravitational action, has to do with maybe less ambitious situations than fully-fledged quantum gravity. Before constructing such theory of quantum gravity on a general background, we should maybe study the situation of quantum matter fields on some fixed spacetime geometry, which may not be flat, but curved due to the gravitational effects. For the moment, we can freeze the gravitation and consider it as a non-dynamical external field present in the system of QFT of matter fields. The theory of quantum fields on curved backgrounds is well studied and well understood. This is that less ambitious idea: let us first quantize matter and put it on a classical background, and for the moment do not attempt to quantize gravity. This situation has its physical importance and significance as well, since all the quantum matter fields have to be treated like, for example, when they are studied on cosmological backgrounds. The Friedmann-Robertson-Walker (FRW) classical background spacetime is a very good approximation to gravitational physics happening some time after the cosmological Planck era. There with good accuracy we can describe effects of the existing gravitational field by putting the quantum theory on curved background. To give an example, we can mention that to get the correct results for big bang nucleosynthesis, which produces all known chemical elements, we must consider effects of cosmological gravitational field, but it suffices that this field be considered completely classical. This approach and motivations first appeared in works on gravitational field by Utiyama and were also repeated recently by Shapiro.

What can quantum field theory of matter fields on curved backgrounds teach us regarding quantum gravity? We may consider the issue with UV-divergences there, when we do not have gravitons on internal lines of Feynman diagrams, but we can have them on external lines emanating from the diagrams. Such semi-classical approach to quantum gravity signifies that we are in a fixed classical external background (external graviton legs correspond to classical interactions with smooth 
external geometry), but at the same time we do not allow gravitons to propagate (not yet) and therefore there are no virtual gravitons in any part of the diagrams. The moral of QFT on curved background is that gravitationally interacting matter fields will generate divergences, for which absorption we will need purely gravitational counterterms constructed with the help of the background external metric field. Of course, these divergences are besides UV-divergences to be found in matter sector, which are roughly the same as the ones we could find in a matter sector, when this one is considered on flat Minkowski spacetime background. (The UV-divergences of the theory probe the coincident limit of two points on the manifold, and the spacetime and internal space too are effectively flat for them in this limit). Thus, let us analyze these new divergences, which have to be absorbed by generally covariant expressions constructed with the background metric. Let us consider a situation, in which the original matter theory is a renormalizable two-derivative theory and without any dimensionful coupling, if it was studied around flat spacetime. For simplicity, we will below focus on the situation at the one-loop level, but the same can be generalized to any loop order. For the case of one-loop off-shell divergences, we find using the formalism of QFT on curved background that the "gravitational" divergences are proportional to the Lagrangian densities:

$$
\sqrt{|g|} R^{2} \text { and } \sqrt{|g|} C^{2}
$$

where $R$ denotes the Ricci scalar and $C$ the Weyl tensor. We have the explicit expression for the second counterterm in four spacetime dimensions (using other more common curvature tensors):

$$
C^{2}=R_{\mu v \rho \sigma} R^{\mu v \rho \sigma}-2 R_{\mu v} R^{\mu v}+\frac{1}{3} R^{2} .
$$

Of course, these two counterterms are non-vanishing only on non-trivial gravitational backgrounds and that is why in flat spacetime theory of matter sector we never had to worry about them. They, however, arise on a curved background (like this one given by the cosmological setup earlier, but there we have $C=0$ on FRW). These are divergences, which should be absorbed by the terms constructed with the external metric, and we see explicitly that they contain four derivatives of the external metric tensor. This is easy to understand since, if the matter theory is without any dimensionful coupling and it is renormalizable in $d=4$ spacetime dimensions, then these "gravitational" divergences must come with dimensionless coefficients and this means that they must multiply terms, which are constructed with precisely $d$ derivatives of the dimensionless metric tensor. In addition, such tensors in four dimensions are precisely $R^{2}$ and $C^{2}$ (when, for the moment, we neglect the third one: Gauss-Bonnet term (GB) and the fourth one: $\square R$, which is a total derivative). Only in this way can we construct necessary counterterms to absorb divergences, which generically show up there in matter theory. We conclude here that, in general, if we are in $d$ dimensions, the divergences generated by the matter sector on a curved background contain exactly $d$ metric derivatives, even if the original action that we would like to use to study propagation of gravitational field contains only two derivatives (and then it is a simple generalization of the Einstein-Hilbert action in general dimension). To absorb these divergences, we need gravitational counterterms with precisely $d$ derivatives. We notice also that these new divergences with $d$ derivatives are induced due to the quantum matter corrections, even if the matter theory contains only two derivatives (or generally less than $d$ derivatives).

Let us recapitulate how to construct higher derivative theories in general dimensions $(d>2)$. The reason why the physical theories have to be higher derivative (HD) theories is simple: the observed dimensionality of spacetime is not 2 . If this was exactly the case seen for macroscopic spacetimes, then two-derivative E-H theory would be enough (and such theory is renormalizable in $d=2$ dimensions). Instead, we see higher dimensionality of physical spacetimes and with the above motivations this leads us to considerations of HD theories. The proper HD theories in $d$ dimensions must be constructed from terms with precisely $d$ derivatives on the metric tensor. For this, we can use various 
curvature tensors (Riemann, Ricci tensors or Ricci scalars) and we can act with covariant derivatives on them. The construction is not restricted only to the $\frac{d}{2}$ powers of the Weyl tensors, like it was above for the on-shell divergent parts of the effective action at higher loop orders in the non-renormalizable E-H theory. In the result, we get some new actions with some new HD terms. Moreover, the coupling parameters in front of these terms are all dimensionless in a general dimension $d$.

The first example of a higher derivative gravitational theory was the theory proposed in 1977 by Stelle $[16,17]$. Its action (in four dimensions) reads:

$$
S_{\text {grav }}=\int d^{4} x \sqrt{|g|}\left(\kappa_{4}^{-2} R+\alpha_{R^{2}} R^{2}+\alpha_{C^{2}} C^{2}\right)
$$

where the coefficients $\alpha_{R^{2}}$ and $\alpha_{C^{2}}$ are dimensionless. This last fact is peculiar to spacetime of dimensionality four, but the structure of the fundamental four-derivative action in general dimension is only modified by one additional term according to

$$
S_{\text {grav }, d}=\int d^{d} x \sqrt{|g|}\left(\kappa_{d}^{-2} R+\alpha_{R^{2}} R^{2}+\alpha_{C^{2}} C^{2}+\alpha_{\mathrm{GB}} \mathrm{GB}\right),
$$

where GB denotes the Gauss-Bonnet term, and again $\alpha_{\mathrm{GB}}$ has the same energy dimensions as $\alpha_{R^{2}}$ and $\alpha_{\mathrm{C}^{2}}$. In four dimensions, the last added term contributes nothing to the dynamics since its variation (to any order) is a total derivative, hence it has no impact on the equations of motion (EOM), nor on the propagator, etc. In higher dimensions, this term contributes to the variations (for example in Lovelock gravitational theories); however, it still contributes nothing to the propagator of graviton around flat spacetime in any dimension. This theory is a first example of a multiplicatively renormalizable gravitational theory in $d=4$ spacetime dimensions. This is an effect due to an improved behaviour of the propagator, which now falls in the UV limit not like $k^{-2}$ (which was the case for the E-H theory with two derivatives), but like $k^{-4}$ and this is really the consequence of having more derivatives in the gravitational action. The increased power exponent on the propagator is essential for having renormalizability because now the energy scaling in the diagram of every internal line is decreased, so we have lower bounds on the superficial degree of divergence of any graph (more about this also later). However, we remark here that this theory is only renormalizable in four dimensions because only there the coefficients $\alpha_{R^{2}}, \alpha_{C^{2}}$ and $\alpha_{\mathrm{GB}}$ are dimensionless. (In higher dimensions, the first renormalizable theory must contain terms with more derivatives). Due to renormalizability, the UV behaviour of Green functions is improved, but still there are infinities and the theory is merely renormalizable (similar like $F^{2}$ gauge theory in $d=4$ is renormalizable), but our goal is to find a candidate for UV-finite gravitational theory (similar to $\mathcal{N}=4$ SYM theory in $d=4$ ). This is a step in a good direction and with it we write gravitational theory in a very similar way to the theories of other interactions present in the SM of particle physics. Now, both the matter and gravitational theory are put on the same footing as theories quadratic in corresponding field strengths or curvatures. We add that the term $\kappa_{d}^{-2} R$ in the action Equation (14) was left there in purpose, although it contains only one power of the gravitational curvature. We keep it for the correspondence with Einstein's gravity in the low energy (that is in the IR) limit of the theory. This is not only a limiting theory, when the derivatives of the metric tensor are small in some sense, but also when the gravitational curvatures are small. We could also include a cosmological constant term $\lambda$ in the action, which contains no derivatives and no curvatures, and hence it would be the first one we have to write in the EFT paradigm for gravitational theories. For completeness, in a theory in $d$ dimensions, we should include, in principle, all terms with up to $d$ derivatives of the metric, if they are not excluded by any symmetry of the theory. They are needed for good IR limits of HD theories. Einstein-Hilbert and the cosmological term are therefore often necessary for a HD gravitational theories in $d=4$.

However, with the advantages of four-derivative theories, we are also meeting some drawbacks here. On the classical level, there are new solutions compared to solutions in standard two-derivative theory. The closer look at them shows that they are typically runaway solutions, hence quite unwanted 
(but compare with an eternal cosmological inflation, which is actually a runaway solution in $R+R^{2}$ Starobinsky theory). On the other side, we remark that in the first four-dimensional renormalizable theory Equation (13), all Ricci-flat spacetimes are also exact vacuum solutions, similarly to the situation of vacuum in the E-H theory. The presence of higher derivatives signifies also the so-called Ostrogradsky instabilities of the theory. This has to do with instabilities of the classical exact solutions of the theory, when written in the Hamiltonian formalism.

The negative sign of the "massive" term testifies to the negative energy of the corresponding state (due to these negative energies these fields are also called phantoms). Of course, we can reformulate the theory (to change the vectors of state) to provide positive energy for the ghosts, but then the massive pole will have a negative residue and therefore the corresponding norm in the Fock space of states will be negative. Hence, the massive spin-2 particles have negative energy or a negative norm and are therefore non-physical. They cannot appear on external on-shell legs of any diagram. The negative norm states cannot be removed from the physical spectrum and hence unitarity in HD theory is violated. Unlike the Faddeev-Popov (FP) ghost, which cancels with non-physical components of the gauge fields and hence preserves unitarity, the appearance of the massive spin-two ghosts contradicts unitarity. Note that the problem of unitarity is the most difficult in higher-derivative gravity. We noticed that in such model we find a presence of massive ghost states with a negative sign of the residuum in the spectrum. This can be interpreted in another way that the putative state of the gravitational vacuum is very unstable and immediately decays (by quantum corrections this is not immediate, but still very rapid decay) and massive ghosts with negative energies are instead produced. Therefore, this argument we may take as a sign that the putative vacuum that we use in the definition of Fock space for HD theories is not the true gravitational vacuum of the theory and we should look for another vacuum state, which is stable. These are all sides of the same coin, which is the violation of unitarity in such class of theories. Lack of unitarity is the general reason why we do not consider this higher-derivative gravity as the first and the best candidate for the quantum gravity description. At the same time, this theory is a very attractive "toy model" for the "true and fundamental quantum theory of gravity". Of course, the problem with unitarity appears in almost any higher derivative theory, whether in gravitational, gauge, or scalar sector.

It is required that we comment here on the interesting situation regarding the renormalizability in the Weyl square gravity. In $d=4$, the theory is non-renormalizable from two loops on [18-20]. However, at the one-loop level and exploiting conformal background gauge, the theory is renormalizable.

We mentioned that in the search for UV-finite theory the next step is a construction of a super-renormalizable theory. We simply get there by considering the following generalization of the four-derivative Stelle theory (where we understand $\square=g^{\mu \nu} \nabla_{\mu} \nabla_{\nu}$ ):

$$
S_{\text {grav }}=\int d^{4} x \sqrt{|g|}\left(\lambda+\kappa_{4}^{-2} R+\sum_{n=0}^{N} \alpha_{R, n} R \square^{n} R+\sum_{n=0}^{N} \alpha_{C, n} C \square^{n} C\right) .
$$

(In principle, we could also add here the higher order generalization of the Gauss-Bonnet term:

$$
\mathrm{GB}_{n}=R_{\mu \nu \rho \sigma} \square^{n} R^{\mu \nu \rho \sigma}-4 R_{\mu v} \square^{n} R^{\mu v}+R \square^{n} R,
$$

but we notice right away here that it does not contribute to the propagator of graviton around flat spacetime in any dimension, so it will not have any impact on the (super-) renormalizability properties). Such theory was proposed for the first time and studied by Asorey, Lopez and Shapiro in 1996 [21]. The feature of its super-renormalizability we discuss in some details below. Here, we would like to say that this theory enjoys even higher suppression of the graviton propagator in the UV, so the loop integrals are made even faster convergent, hence this super-renormalizability property is not unexpected here. We will see that, for a sufficiently high natural exponent $N$, the UV-divergences only remain at the one-loop level. In such theory, the graviton propagator (and also for all other fields in the gravitational sector, like FP ghosts) scales like $k^{-(2 n+4)}$ in the UV regime. Again here, we find the 
same drawbacks of higher derivative theories, related to "bad" ghosts on the quantum level (not to confuse them with "good" FP ghosts). Now, due to the number of derivatives higher than four in the action, we have the situation that the sign of the mass terms for ghosts alternate and we have some set of massive spin-2 normal particles and massive spin-2 ghostly particles, respectively. Now, we can also understand the meaning of monomial or polynomial behaviour of the theory, since the sums in Equation (15) define two general polynomials in the box (that is a covariant d'Alembertian) variable. The comments about the structure of the perturbative spectrum around flat spacetime apply here as well. The problem of unitarity is still present here and it is not alleviated by using gravitational actions with higher than four derivatives, for any fixed natural $N$.

The unitarity, instead, does not depend on the question of dimension, provided that we are in $d>3$ (to have all the characteristics of the relativistic gravitational field turned on). In principle, one could tell about the unitarity by analyzing the perturbative spectrum of the theory around any background. However, the more refined analysis, which also counts the number of degrees of freedom and their linear stability requires the usage of Hamiltonian formulation of the model [22,23]. Therefore, the latter one can be viewed as a non-perturbative and a fully nonlinear analysis. In Weyl gravity, such perturbative analysis points towards non-unitarity; however, some non-perturbative results seem to support opposite conclusions [24-30], which show that either ghosts do not show up or that they are rather innocent, if present.

The final resolution of the problem with unitarity is given by the next upgrade of theories to fully non-local, so to theories containing an infinite number of derivatives ( $N=\infty$ schematically). All terms in the action of such theories could be collected in a form of non-local form-factors, which can be viewed simply as an analytic functions of the covariant differential operator, for example of the covariant box operator $\left(\square=g^{\mu \nu} \nabla_{\mu} \nabla_{\nu}\right)$. Such theories are called weakly non-local because, in their presentation in the action of a theory, all fields and derivatives in the Lagrangians are taken in the same spacetime point. This is to distinguish them from truly non-local (strongly non-local) theories for which descriptions we would have to use kernel operators and the fields depending on different points of spacetime under the same spacetime volume integral. To handle the latter types of theories is more difficult but possible as well. However, the non-local gravitational theories presented in a weakly non-local form suffice to solve the problem with massive unwanted ghost states. The first non-local theories were constructed by Efimov, Kuzmin, Tomboulis and Krasnikov. For the purpose of studying the most general propagator around flat spacetime, it is enough for us to study the following general theory, given by the following gravitational action:

$$
S_{\text {grav }}=\int d^{4} x \sqrt{|g|}\left(\lambda+\kappa_{4}^{-2} R+R F_{R}(\square) R+C F_{C}(\square) C\right),
$$

where there are present two form-factors of the covariant box operator $\square$, namely $F_{R}(\square)$ and $F_{C}(\square)$. (Again, we can here neglect the non-local Gauss-Bonnet term, which contributes nothing to the propagator around flat spacetime). We want to emphasize that, for the issue of power-counting of UV-divergences, which we are going to discuss soon, we can restrict ourselves to the case of flat Minkowski spacetime background because UV-divergences are independent of the background used to evaluate them, due to the background independence of the theory. In the UV limit, any manifold, which is a background, is viewed effectively as a flat one (UV limit is a coincidence limit of two points). Therefore, the power-counting analysis we will perform on flat spacetime and for this purpose we need the most general expression for the gravitational propagator there. Such expression one can easily obtain from the action above, which is indeed the most general one on the flat spacetime.

The action above Equation (17) is an action of a weakly non-local theory, due to the presence of two non-local functions of the box operator. In general, they should be analytic functions of the box operator, but in principle the case of polynomials is also covered here. Moreover, as proven by Tomboulis [31-33], there exist analytic functions such that around real axis on the complex plane they tend in the UV limit (regime, where the argument of the form-factor is real and takes large values) to 
polynomials. These polynomials we would call UV polynomials of the form-factors. This is a very important discovery in mathematical physics, since it gives rise to theories that can completely avoid the problem of ghosts in the perturbative spectrum, but at the same time take all advantages of the HD theories regarding the control over UV-divergences. Indeed, as can be easily checked on particular examples in such non-local theories, we can have no massive ghosts in the spectrum. The structure of the form-factors is due to this being a bit constrained, but they can be still quite general and in a large class of non-local theories. If we use form-factors due to Tomboulis, we indeed avoid the problems with ghosts, but at the UV limit we get back to HD theories described by polynomial functions, not by general analytic functions. The poles of the propagator giving rise to unwanted massive ghosts would show up, if the expression for polynomial HD theories would be valid at all energy scales. However, this polynomial behaviour is valid only in the UV limit. At all ranges of energies, the exact expression is given by the non-local form-factor, which does not develop any pole on the complex plane, besides the massless spin-2 pole of the standard transverse graviton. Hence, these dangerous poles of the HD limit of the theory are completely avoided, due to the usage of interpolating form-factors. In this way, the unitarity of the theory is explicitly preserved and there is no any problem with violation of it, which was present in almost any truncation of the non-local theory to local HD theories. We saw that in HD theories the occurrence of unwanted massive ghost states is often inevitable. However, in non-local theories, we can easily avoid them. Using non-local theories, which in the UV take back the form of HD theories, we omit ghosts and at the same time we can still have a very good situation regarding UV-divergences. A form-factor, which in the UV regime takes the form of any HD theory is always possible. We emphasize here that, about the UV-divergences, only the UV physics (so the UV limit of the theory) decide, so we could completely separate the two issues: of the renormalizability (control over UV-divergences) and of the unitarity (presence of massive ghosts). That thing was not possible for HD theories and this is the real advantage of non-local gravitational theories. As it is well known, the issue of the spectrum and the presence of massive ghost modes in it is an issue for all energy scales, not only in the UV physics. That is why it was possible to disentangle these two problems of renormalizability and unitarity. In the class of non-local theories, we are able to solve both of them at the same time. We add that, in these theories, not only the spectrum is ghost-free, but also the optical theorem is satisfied and unitarity of the $S$-matrix is proven rigorously to all loop orders too [34-36]. In the perturbative spectrum of the theory, we find only physical transverse massless graviton with two helicities and this is true for both the propagator around flat spacetime as well as around any maximally symmetric spacetimes (MSS). The detailed exposition of non-local theories can be found in the papers [37-55].

In the theories, which in the UV limit are described by some HD theory, we observe the following interesting behaviours in the UV regime. First, the theory is asymptotically free in $d=4$ dimensions similarly to the situation with Yang-Mills theories, therefore this opens a possibility for a unification of gravitational and gauge interactions, since they both have very similar UV behaviour. In such case of HD theories, we also have very good fall-off of the propagator in the UV limit, very good control over UV-divergences and we are on a good path towards UV-finite theories. The quantum loops simply behave very well and in a controllable way.

Now, let us consider in more detail the situation with power-counting of UV-divergences in a theory, which in the UV limit takes the following schematic form:

$$
S_{\mathrm{UV}}=\int d^{d} x \sqrt{|g|} \mathcal{R} \square^{\gamma} \mathcal{R},
$$

where by $\mathcal{R}$ we denote a general gravitational curvature (this could be Ricci scalar, Ricci tensor, Riemann tensor or even a Weyl tensor). We noticed that with this choice in the UV the theory has a monomial asymptotics with the exponent $\gamma+2$ on the box in Fourier space as the kinetic operator between gravitational fluctuations (however, $\gamma$ can be here any positive real number; it does not have to be an integer). If the theory has as UV behaviour the general polynomial or even a finite Puiseux series 
form, then still for the superficial and the worst behaviour of UV-divergences we can take the situation, which is given by the highest monomial (with the biggest exponent) and this exponent is what we above called $\gamma$. Such circumstances we can easily achieve, if all the two form-factors in Equation (17) have the same UV asymptotics. Then, the propagator of all modes of the theory (of gravitons and also of FP ghosts) has the same scaling in the UV, as the first time showed by Modesto [56], and this is given by

$$
\Pi \sim k^{-(4+2 \gamma)} .
$$

In the analysis below, we assume that the graviton field $h_{\mu v}$ and a Faddeev-Popov ghost field $C_{\mu}$ (and $\bar{C}_{\mu}$ for a FP anti-ghost field) are dimensionless and this signifies that for these two propagating fields of the theory we will have the same maximal number of derivatives in vertices as well as in propagators of the theory in the UV limit. This also shows that the power-counting analysis of divergences for the case of quantum gravity in any dimension is much easier than the same analysis attempted at a higher derivative gauge or matter theories. As a direct consequence of what was said above, we see that the maximal scaling in the UV of any vertex of the gravitational theory (which may also contain precisely one FP ghost and one anti-ghost field) is

$$
V \sim k^{4+2 \gamma},
$$

which is exactly the inverse of the scaling of the propagator $\Pi$ from the formula Equation (19) above.

Collecting all above facts, we derive the general expression for the superficial degree of divergence $\Delta(\mathfrak{G})$ of any Feynman graph $\mathfrak{G}$ in the theory. Let us imagine that such a diagram contains precisely $L$ loops (so $L$ integrations over momenta are to be done), $V$ vertices (of any type: gravitational with any integer number of legs bigger than two, or also vertices with precisely two FP ghost fields and any number of graviton legs attached) and precisely $I$ internal lines (which correspond to virtual gravitons or FP ghosts). Actually, here we can simplify discussion considerably by considering only gravitons, since, for all that regards FP ghosts, they are very similar to virtual gravitons. Their energy dimensions are the same and they have the same UV scaling of the propagator and the same scaling of any vertex. Let us then, from now on, write only about gravitons, since adding FP ghosts to this analysis will not change anything. For any graph $\mathfrak{G}$, we have the following expression for $\Delta(\mathfrak{G})$ :

$$
\Delta=d L+V[\text { vertex }]+I[\text { propagators }] .
$$

Since, as emphasized above, we have

$$
[\text { vertex }]=-[\text { propagator }]=4+2 \gamma
$$

and taking also the topological relation for any diagram $(I-V=L-1)$ into account, we can write the final bound for the superficial degree of divergence, derived in any dimension $d$, as

$$
\Delta \leqslant d-(2 \gamma+4-d)(L-1) .
$$

Now, let us particularize to the case of $d=4$. There, we get the following expression for the bound on the superficial degree:

$$
\Delta(\mathfrak{G}) \leqslant 4-2 \gamma(L-1) .
$$

Some immediate observations based on this power-counting are here in order. When we tend to increase the exponent $\gamma$ on the box in the action Equation (17), then we see that the UV behaviour given by $\Delta(\mathfrak{G})$ is better and better regarding higher loops with $L>1$. We see that, for some sufficiently high value of the parameter $\gamma$, we have the degree $\Delta(\mathfrak{G})$, which is negative and this signifies by the token of power-counting analysis in the cut-off $\Lambda$ that there are no divergences in the theory at the given loop order. However, we see that always at the one-loop level we find divergences, no matter how high we increase the exponent $\gamma$. Moreover, we also noticed here that at the one-loop level the superficial 
degree (so the worst situation) of divergences is equal to the dimensionality $d$ of the spacetime, at least when we use the cut-off regularization scheme. Being in $d=4$ spacetime dimensions, we find that, for $\gamma \geqslant 3$, there are no divergences at two loops and onwards. In this situation, we say that the theory is one-loop super-renormalizable, since the one-loop level is the last one at which we see divergences at all. In the same way, the theory for $\gamma=1$ we call 3-loop super-renormalizable in $d=4$ dimensions. If we have UV-divergences only at the one-loop level, then we are in a much better situation in hoping for cancellation of them in a some model of UV-finite theory.

There is already a known approach to QFT, which gives the desired result of QFT without any beta functions. Actually, the condition of vanishing of beta functions is equivalent in the parlance of exact RG (or functional RG termed also as FRG) community to the existence of a fixed point of Renormalization Group flow (RG), which is true at all energy scales. This means that the Wilsonian effective action of a theory should always stay at the fixed point, not only in the UV regime. We look for UV-finite theories such that in the UV regime they meet a non-trivial fixed point (meeting a trivial fixed point is quite of boring, because this is a free theory without any gravitational interactions). This is one of the conditions present in the asymptotic safety programme (AS) for quantum gravitational interactions [57-60].

One of the first papers with explicit construction of UV-finite quantum gravitation with higher derivatives appeared in 1989 by Kuzmin [61]. The idea by Kuzmin was the pioneer one and is still very interesting. It has moreover some relations to models with fractional derivatives [62-64].

As a first element in the last step of construction of UV-finite quantum gravitational theory, we remind how the general structure of perturbative UV-divergences looks at the one-loop level. The divergent part of the effective action at this level can be written in a form:

$$
\Gamma_{\mathrm{div}}=\int d^{4} x \sqrt{|g|}\left(\beta_{R^{2}} R^{2}+\beta_{C^{2}} C^{2}+\beta_{E} E\right)+\left(\beta_{\square R} \square R\right),
$$

where $E$ above is another notation for the Gauss-Bonnet scalar (since it gives rise to Euler invariant of the four-dimensional manifold) and we have included also an additional term $\square R$, which is clearly a total derivative. One may ask why we need to include that Gauss-Bonnet term and the last one to the divergent effective action at the one-loop level. Actually, it is perfectly true that they do contribute nothing to the EOM of the effective theory (to their divergent part at one loop); however, the inclusion of such two terms, which after variations are total derivatives, is useful, when we want to discuss the general symmetry properties of the theory. This is important because, when we want to perform some symmetry transformations to check the invariance of the action, then these terms may be essential in providing the correct answer as it is, for example, in some cases in supersymmetric theories. However, here we will be mainly interested in conformal symmetries of the theory and for them the expression above gives rise also to the correct and complete expression for the conformal anomaly of the theory, which we will discuss here in short. All terms in the action above contributes to the conformal anomaly and forgetting about one of them would spoil the derivation related to conformal symmetry at all.

To kill these divergences, we will use killers. What are killers? In the construction of a higher derivative theory (which could be understood as a viable UV limit of some non-local theory with form-factors) so far we have considered only operators, which were quadratic in field strengths (gravitational curvatures). However, it is possible to add also other operators, if they do not change the renormalizability and super-renormalizability properties of the theory. For this to be true, we need the number of derivatives on the metric in additional operators to be smaller or equal to the number of derivatives, which are in terms quadratic in curvatures, which completely determine the form of the propagator on flat spacetime. Then, the renormalizability (and super-renormalizability too) is safe and moreover we know, which operators in the action contribute to the flat spacetime propagator. (Of course, in the full non-local theory, we can add non-local killer terms to the action that only in the UV regime attain the form of higher-derivative terms, which respect the renormalizability, and only in this limit have the number of derivatives bounded by the one appearing in the inverse of the graviton 
propagator in the UV limit). The killer operators have an impact on the beta functions of the theory, but not on the graviton propagator around flat spacetime. They are among the other group of terms, which still could be added to the action and this change does not modify super-renormalizability properties of the theory. One can also speak about terminators and spectators. The terminators participate in killing the beta functions of the theory, but they also modify the propagator; however, the way they are written hides their true nature because they do not look like terms obviously quadratic in gravitational curvatures. The last criminals, which appear at the crime place (of killing the beta functions), are spectators. These operators, still allowed by super-renormalizability, do not contribute neither to the propagator, nor to the beta functions of the operators considered above. They will surely contribute to the finite terms of the effective action at one-loop, but for study of divergences we do not see any effect of them.

Focusing on killers, now we can easily understand that they can be at most quartic in curvatures in four dimensions. This is a corollary of the fact, how the expression of the effective action at one loop looks in an abstract way

$$
\Gamma^{(1)}=\frac{i}{2} \operatorname{Tr} \ln \frac{\delta^{2} S}{\delta \phi^{2}},
$$

where an important fact is that the functional trace denoted above by $\mathrm{Tr}$ is a linear operation and we will expand the natural logarithm according to the power series:

$$
\ln (1+x)=\sum_{i=0}^{+\infty}(-1)^{i+1} \frac{x^{i}}{i}
$$

Since we know that the ansatz for the divergent part of the effective action in four dimensions has the structure of terms, which are at most quadratic in gravitational curvatures Equation (25), then we easily understand by the argument presented above that the terms which are quintic and higher in curvatures will not contribute anything to this divergent action $\Gamma_{\mathrm{div}}^{(1)}$. The killers can be at most quartic in curvatures. (This result depends on the dimensionality of spacetime and in general dimension $d$ the killers for one-loop beta functions may contain up to $d+2$ covariant curvature tensors). Therefore, they can be cubic or quartic in curvatures, while at the same time they may contain any even number of covariant derivatives acting on these curvatures (but these terms as whole must respect the renormalizability criterion from the above).

Due to the mentioned linearity, let us choose to use quartic killers only and use the following addition to the original action:

$$
\begin{aligned}
S_{\text {kill }}= & s_{1} R^{2} \square^{\gamma-2} R^{2}+s_{2} C^{2} \square^{\gamma-2} R^{2}+s_{3} E \square^{\gamma-2} R^{2}+ \\
& +\left(s_{4}(\square R) \square^{\gamma-2} R^{2}\right) .
\end{aligned}
$$

It is easy to understand how our three (four) killers are constructed. We took each term in the ansatz for the divergent part of the effective action at the one-loop level in Equation (25) and simply multiplied them by the scalar expression $\square^{\gamma-2} R^{2}$, where the box operator is understood to act on the square of the Ricci scalar on the right. We also multiply each term by a suitable coefficient $s_{i}$, whose energy dimension is the same of the coefficients $\alpha^{\prime}$ s with the highest $N$ appearing in the quadratic part of the higher derivative action Equation (15). This also ensures that the killers in Equation (28) are not in conflict with renormalizability of the whole theory. There is a mentioned advantage of using quartic over cubic killers in four spacetime dimensions because in the final contributions for beta functions the coefficients $s_{i}$ appear only linearly.

Indeed, in bigger generality, the expressions for all beta functions of the theory we write in the following form:

$$
\beta_{i}=v_{i}+a_{i j} s_{j}
$$


This displays a linear structure of the system of the beta functions of the theory. The matrix $a_{i j}$ is some real constant matrix. The contributions collectively put in $v_{i}$ denote all contributions to the beta functions from all other terms different than four selected killers in the action (from possibly other killers, possibly also cubic and also from terms quadratic in curvature in the action). These are all contributions independent on the coefficients $s_{i}$ of our four chosen killer terms Equation (28). The second term in the above sum in Equation (29) is obviously linear in the coefficients $s_{i}$. Now, the question arises, but it is simple to answer: how we can make our theory so special that it will have no beta functions at all? This is equivalent to demanding from the theory to be so fine-tuned that it is conformal on the quantum level. The condition for UV-finiteness is simply

$$
\beta_{i}=0
$$

The answer is a matter of simple algebra, since we can always find solutions for unknown $s_{i}$ coefficients, if the $v_{i}$ contributions are all given and if the matrix $a_{i j}$ is non-degenerate. This is after all a system of linear equations and, provided that the matrix $a_{i j}$ is non-degenerate, we can always find a real solution for the unknowns. The non-degeneracy of the matrix $a_{i j}$ can be easily grasped as follows. This is the result of the detailed computation of the second variation of these terms with respect to gravitational fluctuations. As from the structure of what is left after stripping $R^{2}$ from these terms, one can see that there is a contribution to each of the terms present in the divergent part of the effective action Equation (25).

Now, the solutions to the linear system of equations Equation (29) always exist and we denote it collectively as coefficients $s_{i}^{*}$. According to what was said above the HD theory:

$$
\begin{gathered}
S_{\mathrm{UV}-\mathrm{fin}}=\int d^{4} x \sqrt{|g|}\left\{\alpha_{R} R \square^{\gamma} R+\alpha_{\mathrm{C}} C^{\gamma} \mathrm{C}+\right. \\
\left.+s_{1}^{*} R^{2} \square^{\gamma-2} R^{2}+s_{2}^{*} C^{2} \square^{\gamma-2} R^{2}+s_{3}^{*} E \square^{\gamma-2} R^{2}+\left(s_{4}^{*}(\square R) \square^{\gamma-2} R^{2}\right)\right\}
\end{gathered}
$$

is completely UV-finite in four dimensions for $\gamma \geqslant 3$ and for precisely tuned values of the coefficients $s_{i}^{*}$, which are the results of solving the system of equations from above Equation (29).

In the example above, we have found a candidate theory for fundamental Quantum Gravity, which is a UV-finite theory. Hence, it is also conformal on the quantum level. Since there are no divergences beyond the one-loop level, in the theory, there are no divergences at all and conformality is completely preserved by quantum corrections. The achievement above is equivalent to finding a theory, in which conformal anomaly to all loop orders is completely absent. We showed explicitly that, at the one-loop level, there are no UV-divergences and the theory is with all beta functions set to zero. In QFT, the trace of the energy-momentum (pseudo-)tensor of the system derived from the effective action is proportional to the beta functions of the theory, or in other words to the conformal anomaly of the theory. We have explicitly in formulas:

$$
T=g^{\mu \nu} T_{\mu v}=\sum_{i} \beta_{i} \mathcal{O}_{i}
$$

where the full action of a system we write as

$$
S=\int d^{d} x \sqrt{|g|} \sum_{i} \alpha_{i} \mathcal{O}_{i}
$$

and the beta functions $\beta_{i}$ are the logarithmic derivatives with respect to energy scale $k$ of the running coupling coefficients $\alpha_{i}=\alpha_{i}(k)$ (some of them run, not necessarily all). The set of operators $\mathcal{O}_{i}$ consists of all generally and gauge covariant operators that we want to add in the Lagrangian of the theory. They have to respect all symmetries assumed from the theory on the classical level. The non-vanishing of the trace on the quantum level of the energy-momentum tensor is called a quantum trace or conformal anomaly. It arises in theories which are conformally-invariant on the classical level and for 
which the process of quantization produces scale-dependence of couplings and in the result quantum trace is non-zero. It is well known that, for normal theories, conformal invariance on the classical level is just an accident and it is generically removed by quantum corrections, which force couplings to run (like for example in YM theory). The theory must be very special to avoid conformal anomaly. However, since in the standard framework of QFT we know how to live with theories that predict and indeed exhibit RG running, then typically this non-vanishing anomaly is not problematic.

For gravity, the situation is diametrically different, since we want to gauge this symmetry (make it in a local version). Therefore, making conformal symmetry non-anomalous is very crucial in construction of a good quantum gravity model. In our case, the physical system is a quantum self-interacting gravitational field. Hence, we cannot truly speak about the energy and momentum of gravitational field here, since they are not generally covariant notions in E-H general relativity. However, some covariant parts of the energy-momentum pseudo-tensor of relativistic gravitational field could be still defined and transform well in some higher derivative gravitational theories [65]. This is why in the pure gravitational setup we should speak only about the trace of the energy-momentum pseudo-tensor.

The trace of the energy-momentum pseudo-tensor of the gravitational field at one-loop level in the gravitational theory in four dimensions off-shell $[1-3,66,67]$ takes the following simple form:

$$
T=\tilde{\beta}_{C^{2}} C^{2}+\tilde{\beta}_{E} E+\tilde{\beta}_{\square R} \square R,
$$

where all three coefficients $\tilde{\beta}_{i}$ are linear combinations of the original beta functions $\beta_{i}$ of the theory as expressed in the divergent part of the effective action in Equation (25).

Here, we eventually see why it is important to keep the last two terms in the divergent part of the effective action Equation (25). The expression for the trace anomaly is not under a spacetime integral; hence, we cannot integrate by parts and we cannot neglect terms with total derivatives here. The last two terms are generally non-vanishing on curved background manifolds and should be included for the proper account of the conformal anomaly. However, in our finite theory, we took care of them too (by adding two suitable killers for $\beta_{E}$ and $\beta_{\square R}$ ). By killing all four beta functions in Equation (25), we also set to zero any linear combination of these coefficients, so, in particular, the combinations appearing in Equation (34). In the result, the total trace of the gravitational pseudo-tensor vanishes in finite theories. This is another check of conformal consistency achieved on the quantum level.

Finally, it is necessary here to mention the following situation happening in UV-finite theories based on super-renormalizable gravitational theories. Such theories, as exemplified by the action Equation (15), contain dimensionful parameters in their higher derivative parts (we neglect here the issue with the presence of the Newton's and the cosmological constant, although they are obviously dimensionful too). This signifies that on the classical level such theory cannot be scale-invariant and exhibits a classical contribution to the trace anomaly $T_{\mathrm{cl}}$ of the gravitational energy-momentum pseudo-tensor. This contribution $T_{\mathrm{cl}}$ is related to the presence of all these dimensionful couplings, and not to their RG running, since there is no RG running on the classical level of the theory. Now, inclusion of quantum corrections adds a new quantum contribution to the anomaly $T_{q}$. What we denote in the formula Equation (34) is a total contribution to the conformal anomaly $T=T_{\mathrm{cl}}+T_{q}$. We see that in UV-finite gravitational theories, the total trace vanishes, or in other words the contributions from quantum loops (which are true RG effects) $T_{q}$ cancel classical contributions $T_{\mathrm{cl}}$ spoiling scale-invariance on the classical level of the theory. This interplay between classical and quantum effects is a characteristic feature of HD UV-finite gravitational theories. Moreover, here we have a cancellation of tree-level classical features not only by one-loop quantum effects but by all loop quantum effects, since the theory for $\gamma \geqslant 3$ is one-loop super-renormalizable. That is, this effect and the virtue of UV-finiteness cannot be reverted by a situation at the two-loop level.

Concluding, we are able to write local conformal symmetry as a consistent symmetry of the gravitational interactions on the quantum level. The fact that all the beta functions vanish we can see as a reason for scale-invariance of all Green functions (for any number of legs, on-shell and off-shell, 
as well as for any number of loops). This determines that the dynamics of the theory is indeed governed by the conformal symmetry on the quantum level. Just obtained Conformal Quantum Gravity stays always at the non-trivial fixed point of the RG. In addition, on the other hand, in this Quantum Gravity, there are completely no divergences, so the problem with infinities is finally solved and the theory fulfills old expectations from the old time, when QFT was born. The long story of infinities is finished.

\section{Conformal Quantum Gravity}

Having constructed a first viable candidate theory for higher derivative UV-finite quantum gravity, we can now come back and analyze in short the question of why we need a conformal field theory (CFT) of quantum gravity. Besides the obvious enhancement of symmetries of the theory and their preservation on the quantum level, we notice that now this model of UV-finite theory is amenable for answering any problem previously associated with quantum gravity. This theory possesses a very good behaviour at the high energy scales (UV limit) and, due to the non-local structure of the theory, this good behaviour is extended to any other scale, and for example the theory is ghost-free. As such, this CFT is a basis for doing conformal perturbation theory, when some operator(s), which is not conformally invariant, is added to the theory and causes the RG running. We know that, in the real world, conformal symmetry must be broken and this is one way that this breaking can be achieved: by adding some non-trivial operator constructed from CFT data as a perturbation to the fixed point behaviour. The symmetric phase of the theory with unbroken conformal symmetry is the basis for studying of various deformations.

The problem of coupling matter to CQG is also interesting. We would like to shortly remark here that quantum aspects of conformal higher derivative quantum gravity coupled to conformally invariant matter were studied in [68,69]. In addition to this, the generalization of conformal higher derivative gravitational theories for the presence of scalar was done in [70]. Finally, the $4-\epsilon$ expansion RG technique was applied to conformal $C^{2}$ gravity with conformal matter in [71].

Moreover, in conformal field theory, we have also (trivial) solutions to the unitarity problem because, in such theory, the scattering matrices are not defined and there is no any problem of unitarity violation. This is, when we insist that the situation with asymptotic states must be conformally invariant too. We remind readers here that, by unitarity of the physical theory, we really mean the unitarity of the $S$-matrix in such theory. Hence, in theories, where we are unable to properly formulate the problem of scattering, we cannot speak about the unitarity of scattering. This is, for example, a situation met on non-trivial spacetime backgrounds (like in cosmology), which do not possess asymptotically flat regions. Basically, in the unbroken (symmetric) phase of the conformal symmetry, we similarly cannot properly define asymptotic states. Every trial to define them leads to inevitable breaking of conformal symmetry and this is a breaking by exact solution of the classical theory. If there are no problems with unitarity and the scattering matrix is trivial, then this also signifies that there is no putative breaking of the unitarity bound for the scattering amplitudes. This is, of course, related to the preservation of the full unitarity in the theory. We also discuss the issues with scattering amplitudes in CFT below in the next section.

On the other hand, the condition on the theory to be conformal puts very strong constraints on the theory and the effective action of it. We can say that, for example in $d=4$, this determines the conformal theory uniquely, if there are no mass scales in the theory. In other cases, the constraints enforced by conformal symmetry are so strong that they restrict the possible form of the form-factor in non-local theories. The constraints are not only put on the terms in the effective action, but also on the anomalous quantum dimensions of the operators. Since Green functions of the theory do not show any scale-dependence, then the only non-trivial elements of QFT are these anomalous dimensions of the operators here, since couplings in front of operators cannot run. There is no RG running, which could be read from the divergences of the effective action. On the quantum level of the theory, we only have finite renormalization of couplings and no scale dependence. The bare values of the couplings that we put in the original classical Lagrangian of the theory generically are not the same as constant 
values, which appear in the effective action. This is the only difference between classical and quantum effective action for conformal theories, which are without any massive parameter on the classical level. However, this finite renormalization of couplings has nothing to do with UV-divergences of the theory and it is just a requirement that our theory takes the values of the couplings from the experiment to make a relation to reality. In principle, the bare action with bare couplings has no relation a priori to couplings that we meet in Nature.

Moreover, since there are no divergences in the theory, we do not need to put and add any renormalization scale (typically denoted by $\mu$ ), which is an optional parameter with one mass dimension, which is typically introduced in non-conformal theories on the quantum level for the purpose of renormalization of infinities in the effective action. This is very great news, since the number and the character of the classical parameters of the theory is preserved and there is no addition of this scale $\mu$. Actually, one can look back and easily understand that the big diversity and complicacy of the effective actions that are found at any loop level in non-conformal theories is due to the appearance of this scale $\mu$ and the possibility to construct infinitely many various terms with it in the effective action. When $\mu$ is not needed, then we do not use it for the construction of a quantum effective theory and the effective action must look much simpler. Finally, as mentioned in the introduction, the presence of the conformal symmetry on the quantum level signifies that the quantization procedure is idempotent and that the quantum effective action is uniquely determined and takes the same structural form as the original classical action of a theory.

Let us also make some detour here and analyze and describe a different model (with conformal dilaton field), which explicitly shows that the conformal symmetry is present both on the quantum as well as on the classical level of the action of UV-finite quantum theory. This way, we can make any UV-finite theory manifestly conformally invariant even on the classical level [72]. We need to add a spurious (that is a non-dynamical) additional field to theory, which we can technically call a conformal compensator field $\phi$. The more common name for this field is dilaton, although, for example, in string theory, it plays a slightly different role and there it is a dynamical field. We can achieve this by performing the following substitution in the gravitational part of the effective action, which we treat as a functional of the metric tensor $g_{\mu \nu}$ in general dimension $d$ :

$$
g^{\mu \nu}=\left(\phi^{2} \kappa_{d}^{2}\right)^{\frac{2}{2-d}} \hat{g}^{\mu \nu}
$$

This is reminiscent of how we can perform conformal transformations on the metric tensor $\left(g_{\mu v} \rightarrow \Omega^{2}(x) g_{\mu \nu}\right)$ and what is the energy dimension of the parameter $\kappa_{d}$. We want the field $\phi$ to have the single energy dimension in spacetime of any dimensionality $d$. Moreover, in the formula above, we denote by $\kappa_{d}^{2}$ the inverse of the square of the $d$-dimensional gravitational Newton's constant. The dilaton compensates over the conformal transformations of the metric, if itself it transforms under conformal transformations according to the following rule:

$$
\phi \rightarrow \Omega(x)^{\frac{2-D}{2}} \phi
$$

where here $\Omega^{2}(x)$ is a general parameter of the conformal transformations. The factor $\Omega^{2}$ appears also when the conformal transformation acts on the metric tensor $g_{\mu v}$ with both indices covariant. An action for a UV-finite theory with the metric $g^{\mu v}$ substituted by $\hat{g}^{\mu v}$ as in Equation (35) is not only conformal on the quantum level because all of the beta functions vanish, but also is manifestly conformally-invariant, if written entirely in terms of the metric $\hat{g}^{\mu \nu}$. This is because the metric tensor $\hat{g}^{\mu v}$ is invariant under conformal transformations. Of course, the first condition here is that all perturbative beta functions of the theory vanish at any energy scale. Only under this condition can we perform and only then the dilaton trick works.

In such theory, we have algebraically " $9+1$ " degrees of freedom in the metric field $\hat{g}^{\mu v}$, since the last tenth degree of freedom is taken by the dilaton field $\phi$. This is regarding the algebraic counting of 
degrees of freedom, while the dynamics of the theory is, of course, very different and the total number of degrees of freedom and their character (and spin) is typically lower and maybe different from these algebraic considerations. (To give a basic example: in E-H theory algebraically, we also have 10 degrees of freedom in a symmetric metric field in $d=4$; however, there are less physical degrees of freedom in the dynamics, and they could be identified with only two helicities of the massless graviton particle).

Moreover, as emphasized extensively in other papers on the series of conformal symmetry, this symmetry is crucial in solving the problem of classical singularities of classical gravitational theories. A proper choice of the conformal scale factor $\Omega^{2}(x)$ makes the GR-like singularities gauge-dependent on the conformal gauge. In addition, as we know, in the true physical description of the system, we cannot use information (like invariants, etc.), which do depend on the gauge choice, and which would kill the gauge symmetry transformation of the theory. Since about some singularities of classical gravity theories we conclude by analyzing the classical non-conformal invariants, then these statements have no meaning in truly conformal gravity. In addition, this regards the singularities appearing in the exact solutions of the classical theory, as well as in solutions of the effective theory arising on the quantum level. Conformal symmetry is crucial in making the theory free of any classical pathologies, not only singularities, but, for example, of closed time-like curves (CTC's), which do appear in such vacuum solutions of Einstein equations as for example found by Gödel. This pathology is resolved by conformal scale factors, which make the time lapse of such curve not a conformal gauge-independent observable and hence it effectively means that any causality breaking effect related to such loop does not appear. Effectively, we can say that the conformal factor rescales the time lapse to zero on such curve and being on it we cannot run into any problem. Similarly, use of conformal transformation resolves various topological singular objects and regions of spacetime, like for a singular ring of Kerr classical spacetime. As it was emphasized earlier, the issue of UV-divergences of a theory is intimately, but non-intuitively, related to the issue of classical small-distance singularities of the theory. This correspondence is also very non-trivial because it links perturbative phenomena of quantum theory with highly non-perturbative strong coupling regime phenomena of the classical theory. In its rough structure, this scheme resembles by now very famous gauge/gravity duality.

We also would like to remind readers that, in the real world, the conformal symmetry must be present in the broken phase and this we can achieve in different ways. One of them was previously introduced with adding some non-conformal operator to the action of CFT and therefore causing a non-trivial RG flow. Another possibility is to exploit, so popular in particle physics, spontaneous breaking (SSB) of conformal symmetry. Since here the conformal symmetry is in the local (gauged phase), then the proper mechanism to achieve this is a conformal gravitational analogue of the Higgs mechanism. In this way, we assume that vacuum expectation value of the dilaton field in the state of the true vacuum of the theory is non-zero and this secretly breaks conformal symmetry. Although it is known that the symmetry, which is local is never truly broken, since it is exact and not approximate, just the Fock state of the vacuum of the theory hides some of all possible symmetries of the theory. We achieve this, if we require that in the physical phase we have: $\left\langle\phi^{2}\right\rangle \neq 0$. This condition for the dilaton field introduces a new mass scale to the theory, since the field $\phi$ has the single power of the energy dimension. Such scale was not present in the original action of the theory. It is in all computations regarding the theory in the broken phase because it comes with the choice of the vacuum state. These issues of the breaking of the conformal symmetry are strictly related to the question about the vacuum state in conformal gravitational theories.

Finally, we would like to admit that conformal gravity (both on the quantum as well as the classical level) is a theory, which fully realizes Mach ideas about the spacetime and matter and their interrelations. We remind that Einstein was influenced by Mach; however, in his standard GR theory, he did not include Mach's profound ideas completely. In Einstein's theory, the inertia of particle (and in bigger generality its energy content) does not depend on the constitution and configuration of spacetime out there, away from the point of the actual location of the particle. Conformal gravity is on the contrary very Machian and this idea is fully embodied by the study of conformal transformations of 
the metric. The possibility of freely doing them, since they are symmetry transformations of the theory, is the link which relates actual location of the particle (here) with global structure of the spacetime (out there). Moreover, the ideas of conformal and Machian origin of mass are very influential for the understanding of the Higgs mechanism and the gravitational origin of mass in the SM of particle physics. It is another virtue that conformal symmetry may help in unifying a well defined UV-finite theory of quantum gravitational field with a particle physics and in providing the same theoretical grounds for origin of both gravitational mass (related to gravitational interactions) and inertial mass (related to other interactions in particle physics, and to Higgs phenomena especially). We are hoping that (probably) a full unification of all fundamental interactions due to conformal symmetry is within our reach.

\section{Conformal Symmetry of the (One-Loop) Effective Action}

As we know in quantum field theory, if there are no divergences of the perturbation theory, then there are no perturbative beta functions of running couplings. We want to discuss the situation at the one-loop level only, since at higher loops the super-renormalizability properties of some theories are only conjectured and they are based on power-counting analysis of UV-divergences. Such overall (or naive) analysis may be hampered by the fact that two or more loop integrals have to be done, even in the Euclidean signature. In addition, as it is known from the studies for the Minkowskian signature case, new UV-divergences may show up after doing the second integral and the analysis of powers for these divergences may not give the expected results. However, there is a rescue for super-renormalizability of higher derivatives theories at higher loop orders in Euclidean signature and the general proof (not based on power-counting analysis) can be done using, for example, Batalin-Vilkovisky formalism of quantization. We also remark that the whole notion of super-renormalizability is tightly related to power-counting and, beyond this, it does not make any sense. Therefore, we will focus on the situation at the one-loop level, treating this first term in the expansion in powers of coupling constant as a physically meaningful one. This is also an expansion in $\hbar$-the quantum physics constant. Of course, since we will not have typically an access to a full resummation of all perturbative contributions or even to non-perturbative contributions, such terms in the expansion still provide us with a very useful information about the quantum physics of some quantum field theory models.

In UV-finite theories, we have a special situation. The question is how special. The analysis here will be independent from the particular regularization scheme used to handle with UV-divergences. We can obtain beta functions using dimensional regularization, cut-off regularization or even $\zeta$-regularization on curved spacetimes. Maybe this analysis here will not apply only to exact beta functions obtainable, for example, in Exact Renormalization Group approaches to QFT (because we do not know how to make all such beta functions vanish by our standard tools). Here, we will consider other examples of theories, which are UV-finite at the one-loop quantum level. However, as described in Section 2, the UV-finite gravitational theories that we found there are finite with all loop orders accuracy because they are one-loop super-renormalizable. However, for the sake of simplicity, here we will also study theories of different types, which firmly shows finiteness at the one-loop level. We know that, in these models, there are no divergences and beta functions are zero. What does this imply for one-loop effective action? The answer for the divergent part of this object is very simple. This is zero in finite theories. Again, we emphasize that there is no divergent part of the effective action at one loop. This we can achieve for sure and this is what we have seen so far.

Since beta functions are zero, we can conclude that the conformal symmetry is somewhere there at the one-loop level. We may say very modestly that it is in the divergent part of the effective action, but this is a trivial fact, since this part of the action is identically zero. Thus, of course, it is there and infinitely many of other symmetries are also enjoyed by the divergent part because zero homogeneously always transforms back to zero. What does it mean and what is the significance of this for the full quantum theory at the one loop? Does this mean that the effective action (finite terms) possesses the conformal symmetry or any other symmetry? Such conclusion is obviously too fast and 
seems to be an absurd one. We know that there is also a finite part of the effective action and this part often will not enjoy symmetries in question.

Let us be a bit more clear about this point. There is a lore of QFT that all the symmetries of the divergent action must be also enjoyed by the finite terms of the effective action. However, since there is no a rigorous proof of such assertion, we are here in position to question it. Does it happen always? What if the divergent action is zero, like above? We cannot derive from this that effective action enjoys any symmetry, which may come to our mind. This is not true, as far as we know, for some effective actions in some QFT models. We think that the significance behind this not rigorously stated lore is that, in good QFT models, quantum anomalies should not arise exclusively due to finite parts only. If there is anomaly of some symmetry, which is by the way enjoyed by the classical original action, then it should show up already in the divergent part and cannot be only visible in the finite part. However, this regards the anomaly of the symmetry of the classical action and not a symmetry of the quantum action! Typically, the quantum theory cannot enhance its symmetries beyond the level which was seen classically. Now, with UV-finite theories, the symmetry seems to be enhanced at the quantum level. For sure, there is some enhancement and some new properties of such UV-finite theories, but, for the moment, it is too much to say that there is a conformal symmetry there. We will argue about this also below. However, at least from this paragraph, it is clear that we cannot use the wisdom of QFT blindly, saying that any symmetry enjoyed by the divergent action must be also respected in finite terms.

Having no divergences is quite a special property of the effective action. However, conformal symmetry of the full effective action at one loop seems to be even more special. Here are the arguments. Let us consider first the situation in those higher derivative theories of gravitation, where there are many tensorial terms that we can construct and add to the gravitational action not changing at all very good UV properties of theories.

Let us consider first the term like $\mathcal{R}^{5}$ in four spacetime dimensions added to a first one-loop super-renormalizable theory (with $\gamma=3$ ). The action of a main theory we schematically write as

$$
S_{\text {grav }, 4 D}=\int d^{4} x \sqrt{|g|} a \mathcal{R} \square^{3} \mathcal{R} .
$$

It is obvious (due to Barvinsky-Vilkovisky formalism [73]) that the term $\mathcal{R}^{5}$ does not contribute to divergences of the theory at the one-loop level. This happens, provided that the number of derivatives on metric in such term is not higher than the number of derivatives on metric in the term in Equation (37) that shapes the UV behaviour of the gravitational propagator, so the renormalizability of the theory is not spoiled. If the original theory (without this $\mathcal{R}^{5}$ term) was finite, then adding this term with whatever front coefficient to the Lagrangian does not change this fact about divergences. In addition, for terms containing also covariant derivatives or higher terms in curvature, this the same (in a situation, when also the exponent $\gamma$ is bigger than the minimal $\gamma=3$ ). Simply, such terms are spectators from the point of view of killing the beta functions. Do we really think that the putative conformal symmetry of the effective action could be so robustly independent on values of so many (in principle very many, only constrained by renormalizability requirements) couplings in front of terms like $\mathcal{R}^{5}$ and higher in curvatures? Then, this would not be a very special symmetry but a robust feature! Such is the UV-finiteness though.

We think that conformal symmetry should be more constraining, like it is on the classical level. There are only few examples of classical actions with conformal symmetry and they are constructed only with a really small number of terms of lower order in curvatures. Coming back to the impact of such after-killer operator, it cannot have any influence on the finite terms of the effective action of the form $\mathcal{R F}_{2}(\square) \mathcal{R}$ either. (This is because of the trace of the second variation, which gives the effective action at one loop). However, it can influence the terms of the type $\mathcal{R}^{3} \mathcal{F}_{3}(\square) \mathcal{R}$ and higher in the effective action. These last terms are not in any way linked to the UV-finiteness, but they could be important for a construction of an effective action enjoying full conformal symmetry. 
Actually, following some ideas suggested in FRG framework for RG flows in QFT, we can think of the finite pieces of the effective action as of a series that we have started to build for a conformal theory. The divergences are not there, but this fact is linked to the specific forms of terms of the types $\mathcal{R F}_{2}(\square) \mathcal{R}=O\left(\mathcal{R}^{2}\right), O\left(\mathcal{R}^{3}\right)$ and $O\left(\mathcal{R}^{4}\right)$. However, then, when we include the $\mathcal{R}^{5}$ term to the original action, some terms in the effective action of order $\mathcal{R}^{3}$ and higher have to be modified accordingly because there is an impact of such operator on these parts of the effective action. In addition, now, if originally the effective action (finite pieces) was conformal, then addition of such operator (of the type $\mathcal{R}^{5}$ ) may change something in this series, in order to recover the conformality we may need to readjust (or even to determine) the coefficients in front of $\mathcal{R}^{5}$ terms. In this way, we construct step by step a series giving the finite parts of the effective action, possibly enjoying conformal symmetry, when new higher in curvature operators are added. We cannot exclude now the possibility that this will lead to conformally invariant effective action. However, we are sure that, for the divergent parts in the UV regime (with beta functions), the series truncates after few initial terms and we do not need to go to the next step in reconstructing full UV-finite theory. If the theory is a minimal UV-finite one (without operators of the type $\mathcal{R}^{5}$ ), then for the finite pieces of the effective action at one-loop, we nonetheless have a series of operators $O\left(\mathcal{R}^{2}\right), O\left(\mathcal{R}^{3}\right), \ldots$ - the so called curvature power expansion of the effective action. The coefficients in this series have to be computed sequentially, so again we can think that the series for conformal theory is possible to be constructed, but at the same time it is very uniquely determined.

Now, still in the super-renormalizable theory Equation (37) at one loop, let us concentrate this time on the impact of the operator $\mathcal{R}^{2}$ (here precisely without covariant derivatives), when added to the original action Equation (37). Again, this does not contribute anything to the UV-divergences. However, this time, because it has too few derivatives on the metric, but not because of too big number of curvatures (like it was in the previous case), it does not influence completely UV-finiteness and perturbative beta functions. However, still it influences the effective action (finite terms) at one loop. We can even say to which term it contributes to the term $\mathcal{R F}_{2}(\square) \mathcal{R}$ and possibly higher in curvature too. Again, it can come with whatever coefficient, but finiteness is very robust and independent of it. Do we think that the supposed conformal symmetry will also be so insensitive and will allow for such a big parameter space? Actually, here there is a finite number of operators with such small number of derivatives on metric and small powers of curvatures (for example, the $\mathrm{E}-\mathrm{H}$ term is in such a set). However, if the conformal symmetry would let this happen, then we do have very little constraints on the form of the conformal action. We think it is not the case and conformal symmetry cannot be so robust. Moreover, the term $\mathcal{R}^{2}$ with any coefficient contributing to the effective action will spoil the series of supposed conformal action, that we described and constructed above. (We remind that this series starts with a term of the type $\mathcal{R} \square^{3} \mathcal{R}$ in the original classical action of a theory). If there is a conformal symmetry there, it should constrain the impact of the term $\mathcal{R}^{2}$ somehow, but we do not see any constraint like this on the level of the perturbative beta functions.

Finally, we want to bring the last argument, why the UV-finiteness is so robust, but conformality should not be. This is when we view higher derivative theories only as UV limits and asymptotics of truly non-local theories with some form-factors. For UV-finiteness, only the behaviour in the UV regime matters, so we concentrate on higher derivatives only, like we did in the two previous paragraphs. This UV-finiteness is again completely independent from the structure of form-factors at finite energies. If we achieve UV-finiteness for one asymptotics of form-factors, then this is a robust feature enjoyed by all form-factors with the same UV behaviour. However, still form-factors at the middle energies may be very different and the effective actions (finite pieces) must depend on these differences. However, we notice that there exist some gravitational observables, which completely do not depend on the precise analytic form of form-factors. These are, for example, tree-level gravitational scattering amplitudes [37]. Conformal symmetry should be very special, but it does not constrain form-factors and in this case the possible parameter space is extremely huge because of the choice of possible interpolating form-factors as analytic functions. The UV-finiteness is a very robust and easy to 
achieve property of some higher derivative or non-local models, but conformality should be a very special property, so it still should be very different from UV-finiteness, but should require the former as the first necessary step. In other words, it is very improbable and unlikely that the theory, which is UV-finite, so from quite a huge class, will reveal itself to be a conformal one, which must be a very restricted and a special theory. For general UV-finite theories, this is like a miracle to also already be conformal. These arguments of robustness of finiteness and speciality of conformality should convince us that the two things are very different and that our UV-finite theories are just a first step on the way towards full conformality at the quantum level. In finite theories, a divergent part of the effective action disappears, but the finite terms generically do not enjoy conformal symmetry. They have to be much more constrained to be called conformal effective actions on the quantum level.

Thus, what is the speciality on the level of finite terms behind the UV-finite theories, theories without divergences, theories without beta functions? There is some physically very interesting feature of these theories-there is no running of couplings in these theories, the couplings are always like bare ones. We do not need to do any infinite renormalization of couplings. However, in the theories (typically finite), we have different energy dimensions of couplings. They could be both dimensionless and dimensionful. The lack of RG running is quite special compared to other QFT models, and we are here at a fixed point of RG. However, what is the impact of these conditions on the finite terms in the effective action? In addition, here comes the basic information about ingredients of infinite renormalization in QFT. If there is a running, then we have to use some renormalization conditions and fix some couplings' values at some physical scale $\mu$. For the construction of the effective action, this means that we have at our disposal one more dimensionful parameter-this scale $\mu$. If we do not have RG flow, we do not have this complicacy.

Now, how do we construct various terms of the effective action (finite terms)? We have to compensate the dimensions of various operators (constructed with curvatures and covariant derivatives) by the dimensions of other parameters present in the quantum theory. For example, due to the RG flow, we need to include terms of the type $\log \square$ or even more non-local terms like $\square^{-1}$ in the effective action. As the parameters for our use, we have couplings in front of all operators and here in finite theories we do not have a scale $\mu$, because of finiteness. Is this very constraining? The answer is no. Still, we can construct operators of the type $\mathcal{R F}_{2}(\square) \mathcal{R}$ or $\mathcal{R}^{2} \mathcal{F}_{2}(\square) \mathcal{R}$ and higher in curvature too, by compensating the dimension of various operators with ratios of various couplings of various dimensions. This is a general situation in higher derivative theories or in non-local theories, where there is a scale of non-locality at our disposal to construct such dimensionally viable terms in the effective action. We conclude that a lot of different terms can still appear in the effective action (in finite terms) and UV-finiteness here does not constrain them at all. This is the situation where we have both dimensionful and dimensionless couplings. It is to be emphasized that the operator additions that we had in the examples considered above $\left(\mathcal{R}^{5}\right.$ and $\mathcal{R}$ or similar ones) come with front coefficients, which are dimensionful in $d=4$. Moreover, the front coefficients in the main action Equation (37) are also such. Thus, for these higher derivative UV-finite theories, we are precisely in the situation described above.

However, let us imagine now that one-loop UV-finiteness is obtained in QFT models, where there are only dimensionless parameters. How then we can construct terms in the effective action (finite pieces)? We do not have $\mu$ at our disposal, and we do not have any other dimensionful parameter at the quantum level because the classical couplings of the theory are all dimensionless. Thus, we can really construct only very few operators in the effective action-only those that have the energy dimension equal to the dimension of the spacetime, really very few. We know what this would mean on the classical level: only scale-invariant classical actions are acceptable (a first step towards conformality). In addition, the same would be repeated for the effective action at one-loop-the same construction, but maybe with different coefficients, but always finite number of terms. Comparing the two actions (the original classical and the quantum effective), we see that here we have only a finite renormalizations of couplings, of dimensionless not RG-running coupling constants. This is really constraining an effective action very strongly. For example, for gauge theory in $d=4$, we could 
have only in the one-loop effective action term of the form $\frac{1}{g^{\prime 2}} F^{2}$, if the theory is one-loop UV-finite, while in the classical action we started with, a term precisely of the form $\frac{1}{g_{0}^{2}} F^{2}$, where $F$ is the gauge field strength.

There is a known example of such finite quantum theory (and not only at one loop, but to all loop orders, and also non-perturbatively) called an $\mathcal{N}=4$ SYM. However, at the one-loop level, there are many more examples of theories scale-invariant classically, which are also UV-finite, so their effective action at one-loop is very constrained and simple. To mention one candidate here: simple bosonic gauge theory (YM) with precisely tuned number of charged massless fermions. It is done as a textbook exercise in QFT to find a condition for one-loop beta function of the gauge coupling to vanish in such model, and the solution is simple, and the resulting theory is one-loop UV-finite and one-loop conformal [74]. We remind readers here that the scale-invariance (so no dimensionful parameters and no mass or energy scales) of the action is a necessary condition for classical conformal invariance of such theory, so on a tree-level. Now, we also understand how this works at the loop levels, if the theory is very special like UV-finite, so not possessing any divergences. Since we want to have a conformal symmetry preserved also on the quantum level, then it seems that the necessary condition is to have it also on the classical level in the first place.

For the moment, here we have considered theories without conformal compensators (dilatons). However, if we include it in the construction of the action, then we may not have any dimensionful coupling or parameter in the theory. This would be good news, since this is the first step towards conformality. However, then during the construction of the effective action, we can use various combinations of operators containing this dilaton field, like in the denominators, in form-factors, in logarithms, etc. We remind readers here that the dilaton carries an energy dimension. Thus, again in this situation, the form of the effective action is not very constrained because how can the dilaton which plays the role of the energy compensator be constrained? We are back to the same case of a theory with dimensionful couplings!

We see that we can obtain conformality on the quantum level, only if we start with a classically scale-invariant theory and without dilaton (or when the dilaton field completely decouples dynamically). Only when we have fine-tuning to get UV-finite theories at loop levels can we have this property of conformality preserved at the quantum level. If we have terms with higher derivatives, then generically we do not deal with conformal theories at a one-loop level, despite the fact that we may have UV-finite theories quite easily (with a small level of fine-tuning of only few parameters in the action) because still we have many dimensionful couplings and parameters in the theory. Hence, the one-loop effective action in such theories is not very constrained and this is not a feature exhibited by conformality. Only for theories already scale-invariant on the classical level, UV-finiteness on the quantum loop level implies conformality on these loop levels. In addition, then the effective action is really very constrained by the power of conformal symmetry there.

It seems that only matter theories with a small number of derivatives (like two) can be conformally invariant at the one-loop level. The examples mentioned above support this claim and in $d=4$ we know all classically conformally invariant actions for all fields with a spin $s \leqslant 2$. To have scale-invariant (or conformal) theories on the classical level (tree-level), a two-derivative massless scalar field theory is sufficient, but it can be also with a quartic potential $\phi^{4}$ (like the Higgs potential) and when we want to couple it to curved geometry, we should also add a term of non-minimal conformal coupling $\frac{1}{6} R \phi^{2}$. Fermions should be massless and without potential (so no Fermi interactions). The standard two-derivative $Y M$ action $F^{2}$ is enough to get the classical conformality too. Finally, we can couple all these matter species in the gauge invariant way using only dimensionless couplings (Yukawa couplings are possible). The total gauge theory can be made one-loop conformal by the construction described above (to add fermions in the precise number). To make it two-loop conformal, one also needs to add scalars. In addition, from this level, one discovers that the spectrum is basically the same like in $\mathcal{N}=4$ SYM theory, and since there interactions are also highly constrained (like the spectrum is), then only the (kinematical) spectrum is enough and it decides about finiteness and conformality to all 
loop levels. Actually, in $\mathcal{N}=4$ SYM theory, the conformal symmetry is preserved on the quantum level by the token that the conformal current appears in the super-algebra of the theory, hence it must be conserved perturbatively and also non-perturbatively, and by both classical and quantum dynamics. The condition of conservation of the conformal current is equivalent to the vanishing of conformal anomaly, hence, in this theory, we have conformality all the time.

However, the situation with gravity is not so fortunate in $d=4$, since only $C^{2}$ gravitational action is classically conformally invariant in $d=4$. We can also add $R^{2}$ term, since it is classically scale-invariant, but this term is not conformally invariant though. However, even with this arbitrary addition, it was found that, in pure gravitational theory, we are not able to cancel the beta functions for all gravitational couplings at one-loop, so the theory is not one-loop conformal. Based on Equation (25), we need to cancel all four structures for quantum one-loop conformality. This is clearly improbable, since, after removing the overall rescaling of the schematic action $R^{2}+C^{2}$, we have only one dimensionless ratio parameter and four beta functions to kill. However, when some matter multiplets are added (motivated by supergravity), within the procedure similar to the one described above for the $\mathcal{N}=4$ SYM, then, according to Fradkin and Tseytlin, it can be made UV-finite, so also one-loop conformal because then such theory contains only dimensionless coupling constants. Maybe this can be extended to higher loops and non-perturbatively also. However, then, which supergravity theory is it? What is its symmetry algebra? Is there a place in the super-algebra for local conformal current? Will it be also covariantly conserved whether the conformal symmetry can be understood on the level of this super-algebra, like in the case of $\mathcal{N}=4$ SYM theory? We can leave these questions aside, since this is a domain of higher derivative conformal supergravity. We only discuss the Fradkin-Tseytlin conformal supergravity briefly in Section 6.

Instead, here we propose a gravitational theory without matter, which still may be one-loop conformal, but it must be defined in a dimension higher than four. Let us be in $d=6$-dimensional spacetime and then consider the following schematic gravitational action

$$
S_{\text {grav }, 6 D}=\int d^{6} x \sqrt{|g|}\left(a \mathcal{R} \square \mathcal{R}+b \mathcal{R}^{3}\right) .
$$

This action, as it stands, is classically scale-invariant (it may contain not only Weyl tensor here, but other curvature tensors as well) because, in $d=6$, the coefficients $a$ and $b$ are dimensionless. It does not need to be here an example of classically conformal action, since only scale-invariance of the classical action is a prerequisite for quantum conformal invariance. In $d=6$, this is not a super-renormalizable theory, but merely a renormalizable gravitational one. However, let us concentrate on the one-loop level only. The divergences are there, but they can be cancelled in DR by adjusting the coefficients $b$ of cubic killers schematically denoted by the terms of the type $\mathcal{R}^{3}$ above. The beta functions for divergent covariant terms of the forms $\mathcal{R} \square \mathcal{R}, \mathcal{R}^{3}$ depend quadratically on the $b$ coefficients. However, maybe it is still possible to solve for them, get real values as solutions and adjust them in such a way that the theory at one-loop becomes finite. Then, this is an example of one-loop quantum conformally invariant gravitational theory in $d=6$ spacetime dimensions. For a similar situation, we may have it in eight-dimensional spacetime with the similar action

$$
S_{\text {grav }, 8 D}=\int d^{8} x \sqrt{|g|}\left(a \mathcal{R} \square^{2} \mathcal{R}+b\left(\mathcal{R}^{3}, \nabla^{2}\right)+c \mathcal{R}^{4}\right) .
$$

Here, all the beta functions are linear in the dimensionless parameters $c$. However, naively, the number of the beta functions to cancel is the same as the number of all terms in the above action, and this number is simply greater than the number of possible mutually irreducible terms of the type $\mathcal{R}^{4}$. Hence, it is quite unlikely to find non-zero solutions for the algebraic system of equations for $a$, $b$ and $c$ coefficients. The dependence on $b$ coefficients is quadratic and on $a$ even more complicated, expressed through some rational functions. However, then maybe also this system can be solved for the $c$ coefficients to get one-loop UV-finiteness and hence one-loop conformality. 
Let us here describe the general philosophy behind the construction of minimal conformal theories in any dimension $d$. In a general dimension, scalars and fermions (spin $\frac{1}{2}$ fields) still have to be described by two-derivative actions to be classically scale-invariant. This means that also their conformal dimensions depend on $d$. However, the gauge field potentials always have the energy dimension one (because of the structure of the covariant derivative). Hence, the construction of scale-invariant classical actions for gauge fields is more involved. Basically, in a general dimension, we have to include all terms that are constructed in a covariant way with gauge-covariant field strengths and with covariant derivatives acting on them, with front coefficients that are dimensionless in given dimension $d$. We couple all these fields by using only dimensionless coefficients. The requirement of scale-invariance is the prerequisite for quantum conformality. Of course, in this way, in the gauge sector, we will inevitably have a dynamics described by HD theories. The conformality on the quantum level (recursively at one-loop, two-loop level etc.). we gain by adjusting some values of the dimensionless coefficients present in the action. With some level of fine-tuning we can reach theories, which will be UV-finite and hence also conformal. Probably, the addition of supersymmetries will also help in this task.

The situation with gravitational theories is a bit similar, but there are also some significant differences. As the basis, we again need to use all terms that are with precisely $d$ derivatives on the metric in general dimension $d$. Therefore, the starting point is the action for first renormalizable gravitational theory. All coefficients are dimensionless and we have classical scale-invariance. In the action for $d>4$, typically we also have some killers. If we hope for one-loop conformality, then we need to fine-tune their coefficients. The counting of free parameters and the beta functions is the following. In beta functions, we need to take care of all terms, also of terms which are total derivatives or become such after variation (like the famous GB terms). However, these terms are not important as killers; they are not even spectators because they do not contribute at all to the effective action (even not to finite pieces), since their general variations vanish. Hence, we have more beta functions to kill than the operators that we can use as killers. Moreover, the last argument, referred to also earlier, decreases the number of killers by one because of overall rescaling of the action. This counting explains, why, in $d=4$, we would need to kill four beta functions with only one free coefficient. As we see, unfortunately, it is quite improbable to kill all the necessary beta functions, and only in the highly symmetric cases can we hope for full cancellation. That is why the study of symmetries of the gravitational action is very important.

As a last remark here, we want to put a comment about conformal theories with dilaton. Then, with the conformal compensator, any theory that is UV-finite can be made explicitly conformally invariant. This trick with using compensators is known for any symmetry. As explained above, finiteness is a necessary condition for getting a conformal theory on the quantum level. Now, by adding a dilaton, we check that the effective action (finite terms) transforms covariantly under conformal transformations, and actually the spacetime integral of this action is a conformal invariant. Technically speaking, we have conformal symmetry there. However, what are the physical consequences of such theory with a dilaton, which appears in many places to cancel the energy dimension?

If the theory is conformally invariant (even in the technical way using the dilaton field), then we are entitled to use conformal transformations of the theory and, in this way, for example, we can succeed with the programme of desingularization of classical gravitational field solutions. With dilaton, we can transfer all singular behaviour to an unobservable conformal factor (dilaton field) in such a way that the (conformal) geometry is completely regular. The non-regular behaviour is now shifted to a conformal gauge-dependent quantity (dilaton field) and in the spacetime all particles feel a regular conformal spacetime, and move in it never reaching a tentative singularity. We emphasize that it was important in this programme first to achieve a construction of UV-finite gravitational theory. Then, using the trick with a dilaton, we made the theory manifestly conformally invariant. This let us conformally desingularize all previously singular solutions. For the theory, which is only classically conformally invariant, we cannot succeed with such a programme. The problem with consistency lies 
on the quantum level. Namely, if some putative theory has non-vanishing beta functions, then we cannot consistently introduce the dilaton field and in this way we can never achieve conformality on the quantum level (even in the technical mode, when we exploit the dilaton field). The advantage of our theory is that we can desingularize full quantum gravitational solutions (that is solutions for the EOM coming from the quantum effective action), because we have the theory conformal on the quantum level. Instead, in theories, only classically conformally-invariant (but with a conformal anomaly on the quantum level), we can remove singularities only from exact classical solutions of the theory. However, then one can argue that quantum effects can bring back "bad" singular solutions and destroy the resolution of singularities, which was achieved only on the classical level. In our UV-finite gravitational theory, this is not a problem (because we have a very good control over quantum effects), and we have a theory showing that all solutions are singularity-free.

However, we believe that, unfortunately, in this disguise for the UV-finite gravitational theories with dilaton field, conformal symmetry does not constrain too strongly the physical observables. We saw already that, on the level of finite terms of the effective action, we may have many (infinitely many, in principle) terms with dilaton fields. They are not constrained at all, conversely to the case of quantum conformal theories without dilatons. However, let us speak about more physical quantities, like the $S$-matrix elements.

\section{Scattering Amplitudes in Scale-Invariant Theories at the Tree-Level}

We have the canonical energy dimensions of fields of various spins and in general dimension $d$ :

- $\operatorname{scalar}[\phi]=E^{(d-2) / 2}$, so $d_{s}=\frac{d-2}{2}$,

- fermion $(\operatorname{spin} 1 / 2)[\psi]=E^{(d-1) / 2}$, so $d_{f}=\frac{d-1}{2}$,

- $\quad$ vector $[A]=E^{1}$, so $d_{v}=1$ and

- $\quad$ two-rank tensor $[h]=E^{0}$, so $d_{t}=0$.

These $d_{i}$ are equal to conformal dimensions used for construction of scale-invariant actions with the fields in question in general dimension $d$, as this was done in the previous section. In scale-invariant theory on-shell amplitudes of generalized scattering processes, in which participates precisely $n_{s}$ scalars, $n_{f}$ fermions, $n_{v}$ vectors and $n_{t}$ tensors are denoted by

$$
S\left(p_{1} \ldots p_{n_{s}}, q_{1} \ldots q_{n_{f}}, r_{1} \ldots r_{n_{v}}, s_{1} \ldots s_{n_{t}}, \epsilon_{1} \ldots \epsilon_{n_{f}}, \kappa_{1} \ldots \kappa_{n_{v}}, \lambda_{1} \ldots \lambda_{n_{t}}\right),
$$

where $p, q, r$, and $s$ are momenta of respectively scalars, fermions, vectors and tensors participating. By $\epsilon, \kappa$, and $\lambda$, we denote the polarization spinors, vectors and tensors. (We must have $n_{f}$ to be an even number). Since in the scale-invariant theory there is no any dimensionful parameter (at the tree-level), we have the following asymptotic scaling property of the above amplitude under a uniform rescaling of all momenta: $p_{i} \rightarrow \alpha p_{i}$

$$
\begin{gathered}
S\left(\alpha p_{1} \ldots \alpha p_{n_{s}}, \alpha q_{1} \ldots \alpha q_{n_{f}}, \alpha r_{1} \ldots \alpha r_{n_{v}}, \alpha s_{1} \ldots \alpha s_{n_{t}}, \epsilon_{1} \ldots \epsilon_{n_{f}}, \kappa_{1} \ldots \kappa_{n_{v}}, \lambda_{1} \ldots \lambda_{n_{t}}\right)= \\
=\alpha^{\alpha \rightarrow+\infty} \alpha^{d-n_{s} d_{s}-n_{f} d_{f}-n_{v} d_{v}-n_{t} d_{t}} \times \\
\times S\left(p_{1} \ldots p_{n_{s}}, q_{1} \ldots q_{n_{f}}, r_{1} \ldots r_{n_{v}}, s_{1} \ldots s_{n_{t}}, \ldots \epsilon_{1} \ldots \epsilon_{n_{f}}, \kappa_{1} \ldots \kappa_{n_{v}}, \lambda_{1} \ldots \lambda_{n_{t}}\right)+ \\
+O\left(\alpha^{d-n_{s} d_{s}-n_{f} d_{f}-n_{v} d_{v}-n_{t} d_{t}-1}\right)= \\
=\alpha^{d-n_{s} d_{s}-n_{f} d_{f}-n_{v} d_{v}} \times \\
\times S\left(p_{1} \ldots p_{n_{s}}, q_{1} \ldots q_{n_{f}}, r_{1} \ldots r_{n_{v}}, s_{1} \ldots s_{n_{t}}, \ldots \epsilon_{1} \ldots \epsilon_{n_{f}}, \kappa_{1} \ldots \kappa_{n_{v}}, \lambda_{1} \ldots \lambda_{n_{t}}\right)+ \\
+O\left(\alpha^{d-n_{s} d_{s}-n_{f} d_{f}-n_{v} d_{v}-1}\right),
\end{gathered}
$$


where in the last equality we used the fact that, for tensors (metric field-gravitons), we have $d_{t}=0$. This is a result based on dimensional analysis only. Moreover, this is an asymptotics for the UV regime of the scattering amplitude. Of course, the coefficient in front of this leading term in the UV asymptotics depends on the particular theory. However, there are no more constraints even if the theory has classical conformal invariance. The only constraint on scattering amplitudes comes from scale-invariance, which is of course a prerequisite for the latter. By asking for Green functions with states, which carry energy-momentum in form of $p_{i}$ (so they carry an energy scale), we explicitly break the conformal invariance of the problem. We warn, however, that this is a breaking "by solution", not spontaneous. The asymptotic states correspond here to solutions of linearized EOM because asymptotically all fields are very weak and are there in the form of plane waves solutions $e^{i p_{i} x}$ multiplied by proper polarizations tensors. These are genuine asymptotic states in the scattering problem. These solutions, as in- and out-states, break the conformal symmetry of the vacuum (or the hidden conformal symmetry of the vacuum, if the last theory was in the spontaneously broken phase). For the $S$-matrix, we analyze Green functions with only such asymptotic states, so we cannot have full conformal symmetry in non-vacuum solutions (plane waves).

Instead, if we consider Green functions of vacuum only (like vacuum to vacuum transitions on curved spacetime or on non-trivial non-flat gauge connection backgrounds), then we should still expect the presence of the full conformal symmetry. Conformality should not to be broken there to scale-invariance, and the constraints on such Green functions should be the consequences of the full conformal symmetry of the theory. Here, the token of scale-invariance is the same as doing dimensional analysis. Finally, we want to emphasize that on-shell scattering amplitudes are different than $n$-point functions in any CFT. The latter are constrained strongly by the conformal symmetry. The former depend on the on-shell conditions, so the plane wave solutions necessarily enter, and that is why conformal symmetry is broken "by solution" and only scale-invariance remains and constrains amplitudes. Additionally, we remark that the problems with asymptotic states are the problems with field configurations localized at spatial and temporal infinity, so very far away from the center, where collisions and interactions take place. This long distance means that they are related to IR configurations. It is, moreover, not surprising that the breaking of conformal symmetry by the introduction of in- and out- states is equivalent to the problem of IR (infrared) singularities of the conformal theory.

Therefore, if the theory is tree-level (that is classically) conformally invariant, then there is no further constraints on scattering amplitudes besides those coming from dimensional analysis, and the amplitudes take the general form described above. Of course, the coefficients of the leading term in the UV are very specific, but none can conclude that these coefficients must vanish. In general, even for conformal theories, this does not occur. These facts are confirmed by the study of scattering amplitudes in tree-level scale-invariant theories or even fully conformal on the quantum level, like $\mathcal{N}=4 \mathrm{SYM}$ theory in $d=4$. Actually, the fact that the theory is quantum conformal does not influence anything on the tree-level scattering amplitudes, since the loops never appeared there. The coefficients are very special, but they do not vanish as we know for the cases of 6-gluon (6-particle) scattering in such theory. The result for this scattering is non-zero at tree-level for just pure YM theory (classically scale- and conformally invariant, but not on the quantum level) and also for $\mathcal{N}=4$ SYM theory (fully quantum conformal). Therefore, conformal symmetry does not trivialize scattering amplitudes at the tree-level. They are sufficiently constrained by scale-invariance of the tree-level action. We want to emphasize that, despite the fact that in the consideration of scattering amplitudes we focus on the tree-level here, the same results can be immediately applied to the scattering on the quantum level in conformal theories on the quantum level. This means that the same conclusions apply to the full quantum scattering amplitudes in $\mathcal{N}=4$ SYM theory and also to scattering in UV-finite models of quantum gravity.

There could be some exceptions to the general UV asymptotics as presented above in a big formula Equation (41) for the scattering amplitudes. However, they come because of some other particular 
conditions and are not related to conformal symmetry. Typically, this says that the asymptotics is softer in UV than the one in the general formula (the exponent on $\alpha$ is smaller than the generic one). For example, for scattering of 3 gluons the result is zero, but this is because of parity symmetry of the amplitude (asymptotics smaller than the naive $\alpha^{1}$ in $d=4$, which is based on the formula Equation (41) applied to the pure YM theory). Here, $\alpha$ is proportional to the energy $E$, so we really speak about energy-scaling of UV amplitudes. Another example is a scattering of gravitons in $d=4$ in Stelle theory (with Einstein-Hilbert term), where the amplitude goes like $\alpha^{2}$, not like expected from the general formula $\alpha^{4}$. This is a consequence of the redefinition theorem and the Gauss-Bonnet identity, which are true together only in $d=4$ [37]. The fact that in pure Stelle quadratic theory in four dimensions

$$
S_{\text {grav }, 4 D}=\int d^{4} x \sqrt{|g|}\left(a R^{2}+b R_{\mu v}^{2}\right)
$$

there is no scattering at all on the tree-level is not a consequence of scale-invariance of this theory. It is similarly not a consequence of classical conformal invariance the more particular fact, that in Weyl square gravity, there is no graviton scattering at the tree-level in $d=4$. These facts are simply consequences of the speciality of four dimensions because only there we have together field redefinition and the GB identity at work. As we see, the conformal enhancement of the symmetries of the classical action (to Weyl square theory possessing conformal invariance) completely does not improve the UV behaviour of scattering amplitudes in this class of theories.

To continue with a similar example, but in $d=6$, let us consider a theory schematically written as

$$
S_{\text {grav }, 6 D}=\int d^{6} x \sqrt{|g|}\left(a \mathcal{R} \square \mathcal{R}+b \mathcal{R}^{3}\right)
$$

In such theory, any tree-level scattering amplitude with an even number of gravitons (bigger than 2) will go asymptotically in UV generically like $\alpha^{6}$. Due to the Furry's theorem for quantum gravity (which is motivated by parity symmetry preserved in such theory), we decided not to consider amplitudes with an odd number of gravitons. A classically conformally invariant action in this dimension is given schematically by

$$
S_{\text {grav }, 6 D}=\int d^{6} x \sqrt{|g|} c_{i}\left(C^{3}\right)_{i}
$$

(various contractions of three Weyl tensors; algebra of indices says that there are only two such contraction possibilities). However, in the theory Equation (44), there is no propagator around flat spacetime, only vertices and all the Green functions are already 1PI diagrams (vertices) at the tree-level. This signifies that, for computation of tree-level scattering amplitudes, we do not need to consider Feynman diagrams here with propagators and internal lines. All amplitudes seem to be read from the vertices of the theory only. A naive, and moreover incorrect, application of the token of conformal symmetry here could say that the scattering amplitudes with gravitons at the tree-level should not contain any power of the energy $E$ of gravitons, regardless of the actual number of them participating in the scattering. However, it would be very strange to say that all scattering amplitudes with gravitons are zero, while the vertices are there non-vanishing on flat spacetime, which is why again the conformal symmetry does not constrain anything regarding the scattering amplitudes here. Actually, we cannot speak about on-shell perturbative states here, so there is no S-matrix! (If the "naive" argumentation is accepted that some supposed amplitudes cannot be proportional to $\alpha^{6}$, then conformal symmetry would require all of them to vanish; moreover, all vertices should vanish, so there would be no theory at all at the tree-level).

Instead, in this theory, we have non-trivial classical Green functions with $n \geqslant 3$ (due to vertices) even on flat spacetime, but we do not have any perturbative scattering amplitudes, since there is no propagation of gravitational perturbations. Here, we do not have at our disposal the redefinition theorem, neither GB identity. What we have instead is the fact that theory is higher than second in 
curvature, so there are no well-defined perturbative asymptotic states. There are no asymptotic states because we cannot define a propagator around flat spacetime. Even some attempts with defining asymptotic states of this theory around maximally symmetric backgrounds MSS (like de Sitter and anti-de Sitter) will fail either because the theory is higher than quadratic in Weyl tensor and again the propagator does not exist on any conformal background, which MSS manifolds are example of. Hence, we know that generic amplitudes in such tree-level (classically) conformally invariant theory vanish, but again this is not a direct consequence of conformality, but of other special circumstances. The similar conclusion can be derived for any theory with the gravitational Lagrangian $\mathcal{R}^{3}$ or higher. The same happens for gauge theories higher in field strengths than quadratic, which are respectively conformal in higher dimensions.

It is now obvious that in such conformal theories on the classical level there is no any problem with the unitarity bound, since the amplitudes are very well-behaved in the UV regime. In the theory above Equation (44), there are no amplitudes, in the theory $C^{2}$ in four dimensions amplitudes could be defined, but they also vanish. In Fradkin-Tseytlin, conformal supergravity based on the bosonic gravitational sector in four-dimensional spacetime on the same $C^{2}$ term, the scattering amplitudes of any number of gravitons have very mild UV-dependence. As we know, the results in such theory are valid both on the classical tree-level, as well as on the quantum level, because the theory is quantum conformal. The channels with graviton exchange contribute nothing and we have only Feynman diagrams with mediation of scalars and gluons of $\mathcal{N}=4$ SYM theory. It is well known that the scattering of gravitons due to only virtual gluons and scalars gives rise to the amplitude, which in the UV limit tend to a constant, which is related to the SYM gauge coupling constant. In addition, of course, such a gauge coupling constant does not put a unitarity bound in danger at all. Hence, we see that, in all examples considered here of conformally invariant gravitational theories, the unitarity bound is always satisfied.

The last remark is about the theory with dilaton (conformal compensator). Then, the theory is without any dimensionful parameter, so it is at tree-level scale-invariant. The amplitudes should follow the general formula Equation (41) presented above. However, the dilaton takes the vacuum expectation value (v.e.v.). Thus, this means that in the vacuum of the theory there is a new energy scale, or simply this vacuum is not scale-invariant. We cannot have dilaton on external on-shell legs, but its v.e.v. can be a new parameter, that amplitudes may depend upon. Since the scale-invariance is broken even by the vacuum choice (dilaton v.e.v.), then here the scale invariance is not broken only by solutions, and the derivation above for the general formula Equation (41) does not hold. The UV asymptotics of physical amplitudes is not constrained by scale-invariance and it could be quite complicated. If the dilaton is frozen in its v.e.v. and we cannot have it on external legs, then this is like a theory with many mass scales, so there is no constraint on scattering amplitudes besides the proper energy dimension, that they should have (indeed, this is what the dilaton checks). The dilaton can be also a virtual dynamical excitation, but not the physical one. However, this still does not reintroduce scale-invariance or the conformal invariance constraints on the scattering amplitudes.

In conformal theories without dilaton (these are highly constrained theories too), the scattering matrix elements can depend only in a very precise way on the energy of colliding particles Equation (41). This way is dictated by scale-invariance of the theory. Otherwise, this would violate assumed conformality, which implies scale-invariance. This is the case of one-loop conformal theories, where scattering amplitudes at one-loop are the same (up to a finite renormalization of coupling constants) as the tree-level ones. Thus, indeed, they are very simple and all effects of quantum dynamics are washed away in the structure of these amplitudes.

What about scattering of physical excitations in conformal theories on the quantum level with dilatons? Looking at the effective actions, which generate such amplitudes, we notice that they are not very constrained. They are quite general and have many terms. Thus, what is the role of conformal symmetries embodied here in the form of dilaton? We think, that its effect is quite simple. Basically, it constrains the amplitudes in such a way that they have the correct energy dimensions. No more 
constraints-only the constraint of overall energy dimensions, which we could derive by ourselves easily earlier by dimensional analysis, without the need to employ dilatons and conformal symmetry. This is because the dilaton stays there, where it is needed to compensate for unbalanced energy dimension for some operators. Like emphasized above, the true power of conformal symmetry is to be seen, when the dilaton field dynamically decouples. Moreover, for such scattering amplitudes of physical particles, we cannot have a dilaton as external particle because, by using conformal symmetry, we can always gauge it away. Thus, its presence on the external legs is conformal gauge-dependent. In addition, as a physical excitation, we cannot allow it because it depends on a gauge. This concludes the part about scattering amplitudes in conformal theories with dilatons.

The following part contains some new arguments and understanding of the issues, which were discussed for a long time about finite terms, $\mathcal{N}=4$ SYM theory, effective actions, scattering amplitudes, dilatons and conformal symmetry.

Before, we put two statements about the $S$-matrix in conformally invariant theories. It could be possible to reconcile the two, despite the fact that naively they seem to be contradictory. The first claim was that the $S$-matrix does not exist because one cannot speak about any asymptotic state. The second one is that, when one does computation, there is a formula for scattering amplitude, which is constrained only by scale-invariance. These statements could be both true at the same time and below we explain how this happens.

One must think operationally how one would check the statements above. In both cases, one would have to compute the scattering amplitudes. Imagine that we could do it, for example, with a computer. Then, there is a unique well-defined result, but to do this we had to pick up some form of the asymptotic states. In addition, this act of picking up solutions breaks conformal invariance, while in the conformal theory in question there should be no asymptotic states at all, if there is conformal invariance unbroken, which means that it is unbroken also by solutions, or, in other words, how would one operationally prove that the $S$-matrix does not exist? There could not be any explicit proof of the calculation even for some specific theory. Why?-because then one uses some asymptotic states, so one has broken (maybe accidentally and unawarely) conformal symmetry. Thus, this is a fact, which is not provable by any analytic computation. It is a statement that is to be proven only by asking for the absence of asymptotic states due to the symmetries of the theory.

Now, for our purposes, for energies above the Planck energy, we can think of the phase in which conformal symmetry is in the unbroken state. In addition, then use the second statement that one cannot speak about asymptotic states and that there is no problem with a unitarity bound of the $S$-matrix. There is no $S$-matrix there for $E>M_{\mathrm{Pl}}$ ! One can say that, if someone tries to insist on computing the $S$-matrix in such a phase, then he will break the conformal invariance by the introduction of asymptotic states, so the symmetry is gone, but in the symmetric phase we surely do not want this to happen.

One can ask, in the light of previous comments, what is the meaning of this whole industry of computing amplitudes in $\mathcal{N}=4$ SYM theory-because, if from the beginning the conformal symmetry is used, then the answer should be trivial-there are no asymptotic states, no scattering matrix. In our opinion, the situation is as follows. The $\mathcal{N}=4$ SYM is indeed a very special, conformal theory, without any doubts. However, when people insist on computing tree-level amplitudes, then they reduce its speciality and the conformal symmetry is gone. In addition, they find amplitudes like in any local two-derivative gauge theory with the fermionic and scalar matter. We mean that the result for the structure of these amplitudes is not constrained by conformal symmetry. Of course, for a particular theory, the coefficients in amplitudes are precisely and uniquely defined; however, there is nothing of conformal symmetry there. It is like a scattering in any scale-invariant gauge+fermions+scalars system at the tree-level. The conformal symmetry of $\mathcal{N}=4$ SYM does not kill any term in the amplitudes and likely all possible terms in such a situation are generated. The coefficients in the amplitudes are only special because the coefficients in the Lagrangian of $\mathcal{N}=4$ SYM are very special, but they are not zero. The true power of the conformal symmetry comes only because of the loop level. As we 
know, the total quantum effective action is there identical to the original action with an only finite shift of the YM gauge coupling, there is no divergent part, etc. In addition, to prove this by explicit one-loop computation is quite tedious. However, knowing all tree-level results now in the industry of amplitudes the results of quantum amplitudes could be also firmly and easily predicted. We also note an interesting correspondence between scattering amplitudes in conformal gravity based on $C^{2}$ action and similar amplitudes in YM theories in $d=6[75,76]$.

If in the conformally invariant theory we cannot define $S$-matrix, by construction, then all problems of non-unitary higher derivative theories disappear because the question of unitarity can be only asked, if we can speak about the $S$-matrix. Then, higher derivative quantum conformal theories satisfy all the requirements of the consistent theories for quantum gravity.

\section{Conformal Supergravity}

In the quest for a conformal quantum gravity, we must note that the first successful model was formulated in the framework of extended Weyl square supergravity theory by Fradkin and Tseytlin in 1984 [20,77-81]. The authors used $\mathcal{N}=4$ supergravity theory based on higher derivative action coupled to two copies of $\mathcal{N}=4$ SYM theory. However, they were not able to find full symmetries of the coupled system and they stated that this system was with eight explicitly visible supercharges. One may think that the possible theory with more supercharges would put even stronger constraints, like an $\mathcal{N}=8 \mathrm{E}-\mathrm{H}$ supergravity puts on the dynamics in the gravitational supermultiplets there. The crucial thing in the construction by Fradkin and Tseytlin was the usage of $C^{2}$ action in four dimensions as the action in purely gravitational bosonic sector. This is in distinction to the case of other supergravity models, which were based on two-derivative dynamics given by $\mathrm{E}-\mathrm{H}$ action. The obvious advantage is that such theory is naturally, at a classical level, conformally invariant, while the E-H supergravity is not. The latter contains a dimensionful parameter-Planck's mass. In the former theory (Weyl square gravity), the effective Newton's constant arises at the solution level because all the solutions (except conformally flat manifolds) break the conformal invariance. This is similar to the situation in classically conformally invariant four-dimensional electrodynamics, where the Coulomb potential solution comes with a scale and this solution explicitly breaks conformal invariance of the theory. Since in $C^{2}$ gravity we have conformal invariance at the beginning, it is not difficult to keep it also at the quantum level after coupling it to a sufficient amount of matter, which is superconformal by itself (but on the flat background).

This conformal supergravity theory comes in four dimensions without any mass scale. This is not surprising since scale invariance (absence of mass scales) is a first condition towards conformal invariance on the classical level. It seems that only in four dimensions we are very lucky that we can study scattering processes in conformal theory. Moreover, with Fradkin-Tseytlin theory, in opposition to quantum conformal gravity presented in Section 3 , we have the luxurious situation that the couplings of the theory are dimensionless, so the theory is scale-invariant also on the classical level. In this very interesting theory, the symmetry algebra is so big that the considerations about the effective action from the Section 4 are all true. Basically, the quantum effective action at any loop order coincides in the structure with the classical action (and we could have only finite renormalizations of couplings). This means that this theory is very simple, very beautiful and very constrained on the quantum level.

Fradkin and Tseytlin explicitly computed and showed that all beta functions in such theory vanish to perturbative and also to non-perturbative level as the result of very high symmetry of the theory and precisely chosen spectrum of matter fields coupled to conformal supergravity. In their approach, the conformal symmetry is another gauge symmetry of gravitation, together with diffeomorphism symmetry and with $S U(N)$ gauge symmetries of the matter sector. All these symmetries are local as they should be in a consistent quantum gravity theory. The only apparent problem as the proponents of this revolutionary theory had noticed, was that this theory treated perturbatively to compute scattering matrix elements gives naively non-unitary results. However, the resolution to this puzzle can be also found above in this article. First, as emphasized in Section 2, pure monomial supergravity theory based 
on $C^{2}$ action in the gravitational sector exhibits a propagator with only one pole, but with multiplicity 2 , before a splitting. This is a pole in a spin- 2 sector and without a mass. The ghosts appear when one insists on doing a splitting and a resolution of this double pole into single poles.

On the other hand, since the quantum theory of F-T supergravity is conformal (all quantum beta functions vanish), then strictly speaking and according to arguments presented in the previous section, we should not speak about scattering processes in this conformal theory at all. Thus, all the results obtained by insisting on using asymptotic states bring with them the artifacts of breaking the conformal symmetry. This could explain the first initial observations made by the authors about apparent violation of unitarity in scattering processes. Additionally, we should remind readers that precisely in $d=4$ dimensions we do not have any scattering in the graviton sector, since there we could use the redefinition theorem and GB identity, like this was done already in Section 5 for a general four-dimensional Stelle's quadratic theory. We think that, if supersymmetry is powerful enough, then this result can be also transferred to the matter sector, or could constrain the mutual interactions between matter and gravitation. Furthermore, if the supersymmetry in the local version is powerful enough, then it could transmit the preservation of the unitarity from the gauge sector (where it is clearly there for YM gauge fields) to the graviton sector. Then, the issue with unitarity in this theory would be completely explained and we could view this model as a fully viable candidate for conformal quantum gravity.

There is one additional thing that could be expected from the highly supersymmetric and conformal theory of supergravitation. This is the question of grand unification of gravity and matter fundamental interactions. Unfortunately, in $\mathcal{N}=4$ supergravity as introduced by Fradkin and Tseytlin, the number of supercharges and related supersymmetries is too small to relate matter and gravity sector in a very tight way, which would constitute a true unification of all interactions. Instead, such strong tights are known to exist for $\mathcal{N}>4$ supergravities based on E-H action, where the matter sector and interactions in it are strictly related to what happens in gravity. In the language of algebraic classification of particle representations, the supermultiplet, which contains gravity in such higher supersymmetric theories contains also parts (or whole, like in the $\mathcal{N}=8$ supergravity case) of the coupled supersymmetric matter sector supermultiplets. It is known that in $d=4$ the number of conformal supercharges is constrained and cannot exceed 16, even for higher derivative supergravitational theories [82]. In the theory by Fradkin and Tseytlin, we have eight conformal supercharges. If somehow miraculously the $\mathcal{N}=8 \mathrm{E}-\mathrm{H}$ supergravity is a UV-finite theory, then we should expect there this maximal number of 16 superconformal supercharges (maybe not all of them will be explicit, some maybe realized as hidden symmetries of this theory). This would suggest that we could also try to extend the F-T supergravity for a bigger number of conformal supercharges, like for the situation in $\mathcal{N}=8$ theory. However, such extension is at the moment beyond our reach. We see that, in principle, there is a space for further development of the model originally constructed by Fradkin and Tseytlin.

Finally, we can mention that, in the Fradkin-Tseytlin conformal supergravity, there are two dimensionless coupling constants: the coupling in front of the Weyl square gravitational term $\alpha_{C^{2}}$ and the coupling in front of gauge fields $g_{\mathrm{YM}}$. In principle, they are completely independent constants. In a theory where the unification is successful, there should be a very strict algebraic relation between these two couplings. We could expect such relation as a consequence of supersymmetry transformations mixing matter and gravitational degrees of freedom. In the bigger supermultiplet, we should expect both matter and gravitational fields, and this is also another expression of the unification idea: to put all interacting fields in the same multiplets of the same symmetry group (this may not be a bosonic symmetry, but could be a fermionic one, like a supersymmetry is). However, this is not a situation in Fradkin-Tseytlin supergravity. We expect that a higher conformal supergravity should be able to give clues for such unification of couplings, and matter with gravitation in the same symmetry supermultiplets. 


\section{Conclusions}

In this review, we showed explicitly what were the main problems of quantum gravity formulated in the language of quantum field theory. We also presented a construction of a theory that overcomes all these problems. The final theory is without divergences in the UV regime and without classical singularities for small distances (coincident limits). It was essential to employ conformal symmetry in the local version in the gravitational framework to succeed with these goals. The conformal symmetry of the final theory is present both on the classical and on the quantum level. Technically, we see it manifest, when we use a dilaton field. The theory presents a very controllable behaviour on the quantum level, which is equivalent to vanishing of conformal anomaly. The version of a theory, in which all couplings are dimensionless, was also discussed. Furthermore, we mentioned Fradkin-Tseytlin theory with increased level of supersymmetries in a local version. We presented some new understanding related to the form of the effective actions and scattering amplitudes in classical and quantum conformal field theories. We advocated the virtues and specialities of the known quantum CFT models. It is remarkable that quantum gravity is now among them.

It is obvious that, in the real world, conformal symmetry is broken. However, this does not mean that the formulation of the fundamental theory does not enjoy conformal symmetry on the theory level. It is not known if in the real world the conformal symmetry is broken by the vacuum choice (v.e.v. of the dilaton) or by solutions, which necessarily break it, or it is just an approximate symmetry and it was never exact, or maybe there exists and is realized a still different method of its breaking. In this former case, the world can experience scale-dependence and RG flows due to some operator added to the CFT, which describes an UV fixed point. This perfect CFT with gravitational interactions is a starting point for the conformal perturbation theory. To push forward such a programme, all the CFT data should be known about the operators present in the CFT. We will compute in future the anomalous scaling dimensions of primary (and also of higher generation) operators in this gravitational CFT model. Another point is about the detailed study of SSB of conformal symmetry and the choice of the profile for the dilaton field. We just remark that, contrary to the case of scalars in particle physics, which in the vacuum state can only take constant values; here, the dilaton field can take some spacetime dependent profiles. Besides the issue of breaking of conformal symmetry, we shall also investigate in the future the role of conformal symmetry for the form of exact gravitational solutions. We believe that conformal symmetry constrains them too in a similar way like it constrained effective actions and scattering amplitudes. Moreover, this last issue is related to the possible ways of breaking of conformal symmetry in the real world.

Having constructed the basis and the frame for quantum conformal gravitational interactions, we can now start studies of the issues related to breaking of conformal symmetry and its impact on the gravitational theory. The research on conformal gravity is quite popular and its various aspects are already being investigated. We refer the interested reader for the literature in Refs. [83-90]. It is also of great interest to focus on a possibility of experimental or observational verification of the conformal gravitational theory. Some studies in this direction were already performed in [91-93]. We believe that, due to the high symmetry of the theory and the mathematical beauty present in its construction, the verification that this is the correct theoretical framework for quantum gravitational interactions will come soon.

Acknowledgments: L.R. would like to thank Leonardo Modesto at SUSTech, in Shenzhen, China for warm hospitality and the invitation to the conference "International Conference on Quantum Gravity".

Funding: This research received no external funding.

Conflicts of Interest: The author declares no conflict of interest.

\section{References}

1. Asorey, M.; Gorbar, E.V.; Shapiro, I.L. Universality and ambiguities of the conformal anomaly. Class. Quantum Gravity 2003, 21, 163. [CrossRef] 
2. Capper, D.M.; Duff, M.J. Trace anomalies in dimensional regularization. Nuovo Cimento A 1974, 23, $173-183$. [CrossRef]

3. Capper, D.M.; Duff, M.J. Conformal Anomalies and the Renormalizability Problem in Quantum Gravity. Phys. Lett. A 1975, 53, 361-362. [CrossRef]

4. Duff, M.J. Twenty years of the Weyl anomaly. Class. Quantum Gravity 1994, 11, 1387. [CrossRef]

5. Kallosh, R.; Linde, A.D.; Linde, D.A.; Susskind, L. Gravity and global symmetries. Phys. Rev. D 1995, 52, 912-935. [CrossRef]

6. Banks, T.; Seiberg, N. Symmetries and Strings in Field Theory and Gravity. Phys. Rev. D 2011, 83, 084019. [CrossRef]

7. t Hooft, G.; Veltman, M.J.G. One loop divergencies in the theory of gravitation. Ann. Inst. H. Poincare Phys. Theor. A 1974, 20, 69-94.

8. Van Nieuwenhuizen, P.; Wu, C.C. On Integral Relations for Invariants Constructed from Three Riemann Tensors and their Applications in Quantum Gravity. J. Math. Phys. 1977, 18, 182-186. [CrossRef]

9. Goroff, M.H.; Sagnotti, A. The Ultraviolet Divergences Of Gravity Theories. In Proceedings of the Fourth Marcel Grossmann Meeting on General Relativity, Rome, Italy, 17-21 June 1985.

10. Goroff, M.H.; Sagnotti, A. The Ultraviolet Behavior of Einstein Gravity. Nucl. Phys. B 1986, 266, 709-736. [CrossRef]

11. Goroff, M.H.; Sagnotti, A. Quantum Gravity At Two Loops. Phys. Lett. B 1985, 160B, 81-86. [CrossRef]

12. Bern, Z.; Carrasco, J.J.M.; Johansson, H. Progress on Ultraviolet Finiteness of Supergravity. Subnucl. Ser. 2011, 46, 251-276.

13. Bern, Z.; Carrasco, J.J.; Dixon, L.J.; Johansson, H.; Kosower, D.A.; Roiban, R. Three-Loop Superfiniteness of $\mathrm{N}=8$ Supergravity. Phys. Rev. Lett. 2007, 98, 161303. [CrossRef] [PubMed]

14. Bern, Z.; Dixon, L.J.; Roiban, R. Is N = 8 supergravity ultraviolet finite? Phys. Lett. B 2007, 644, $265-271$. [CrossRef]

15. Banks, T. Arguments Against a Finite $\mathrm{N}=8$ Supergravity. arXiv 2012, arXiv:1205.5768.

16. Stelle, K.S. Renormalization of Higher Derivative Quantum Gravity. Phys. Rev. D 1977, 16, 953. [CrossRef]

17. Stelle, K.S. Classical Gravity with Higher Derivatives. Gen. Relat. Gravit. 1978, 9, 353-371. [CrossRef]

18. Fradkin, E.S.; Tseytlin, A.A. Renormalizable Asymptotically Free Quantum Theory of Gravity. Phys. Lett. $B$ 1981, 104, 377-381. [CrossRef]

19. Fradkin, E.S.; Tseytlin, A.A. Renormalizable asymptotically free quantum theory of gravity. Nucl. Phys. B 1982, 201, 469-491. [CrossRef]

20. Fradkin, E.S.; Tseytlin, A.A. Conformal Anomaly in Weyl Theory and Anomaly Free Superconformal Theories. Phys. Lett. B 1984, 134, 187-193. [CrossRef]

21. Asorey, M.; Lopez, J.L.; Shapiro, I.L. Some remarks on high derivative quantum gravity. Int. J. Mod. Phys. A 1997, 12, 5711-5734. [CrossRef]

22. Kluson, J.; Oksanen, M.; Tureanu, A. Hamiltonian analysis of curvature-squared gravity with or without conformal invariance. Phys. Rev. D 2014, 89, 064043. [CrossRef]

23. Riegert, R.J. The Particle Content Of Linearized Conformal Gravity. Phys. Lett. A 1984, 105, $110-112$. [CrossRef]

24. Tomboulis, E. 1/N Expansion and Renormalization in Quantum Gravity. Phys. Lett. B 1977, 70, $361-364$. [CrossRef]

25. Tomboulis, E. Renormalizability and Asymptotic Freedom in Quantum Gravity. Phys. Lett. B 1980, 97, 77-80. [CrossRef]

26. Kaku, M. Strong Coupling Approach to the Quantization of Conformal Gravity. Phys. Rev. D 1983, $27,2819$. [CrossRef]

27. Bender, C.M.; Mannheim, P.D. Exactly solvable PT-symmetric Hamiltonian having no Hermitian counterpart. Phys. Rev. D 2008, 78, 025022. [CrossRef]

28. Bender, C.M.; Mannheim, P.D. No-ghost theorem for the fourth-order derivative Pais-Uhlenbeck oscillator model. Phys. Rev. Lett. 2008, 100, 110402. [CrossRef] [PubMed]

29. Tkach, V.I. Towards Ghost-Free Gravity and Standard Model. Mod. Phys. Lett. A 2012, 27, 1250131. [CrossRef]

30. Smilga, A.V. Supersymmetric field theory with benign ghosts. J. Phys. A 2014, 47, 052001. [CrossRef]

31. Tomboulis, E.T. Superrenormalizable gauge and gravitational theories. arXiv 1997, arXiv:hep-th/9702146. 
32. Tomboulis, E.T. Renormalization and unitarity in higher derivative and nonlocal gravity theories. Mod. Phys. Lett. A 2015, 30, 1540005. [CrossRef]

33. Tomboulis, E.T. Nonlocal and quasilocal field theories. Phys. Rev. D 2015, 92, 125037. [CrossRef]

34. Briscese, F.; Modesto, L. Cutkosky rules and perturbative unitarity in Euclidean nonlocal quantum field theories. arXiv 2018, arXiv:1803.08827.

35. Christodoulou, M.; Modesto, L. Reflection positivity in nonlocal gravity. arXiv 2018, arXiv:1803.08843.

36. Asorey, M.; Rachwal, L.; Shapiro, I.L. Unitary Issues in Some Higher Derivative Field Theories. Galaxies 2018, 6, 23. [CrossRef]

37. Dona, P.; Giaccari, S.; Modesto, L.; Rachwal, L.; Zhu, Y. Scattering amplitudes in super-renormalizable gravity. J. High Energy Phys. 2015, 2015, 38. [CrossRef]

38. Modesto, L.; Piva, M.; Rachwal, L. Finite quantum gauge theories. Phys. Rev. D 2016, 94, 025021. [CrossRef]

39. Modesto, L.; Rachwal, L. Exact solutions and spacetime singularities in nonlocal gravity. J. High Energy Phys. 2015, 2015, 173.

40. Giaccari, S.; Modesto, L.; Rachwal, L.; Zhu, Y. Finite Entanglement Entropy of Black Holes. Eur. Phys. J. C 2018, 78, 459. [CrossRef]

41. Koshelev, A.S.; Modesto, L.; Rachwal, L.; Starobinsky, A.A. Occurrence of exact $R^{2}$ inflation in non-local UV-complete gravity. J. High Energy Phys. 2016, 2016, 67. [CrossRef]

42. Modesto, L.; Rachwal, L. Finite Conformal Quantum Gravity and Nonsingular Spacetimes. arXiv 2016, arXiv:1605.04173.

43. Bambi, C.; Modesto, L.; Rachwal, L. Spacetime completeness of non-singular black holes in conformal gravity. J. Cosmol. Astropart. Phys. 2017, 2017, 3. [CrossRef]

44. Bambi, C.; Modesto, L.; Porey, S.; Rachwal, L. Formation and evaporation of an electrically charged black hole in conformal gravity. Eur. Phys. J. C 2018, 78, 116. [CrossRef]

45. Modesto, L.; Rachwal, L. Universally finite gravitational and gauge theories. Nucl. Phys. B 2015, 900, 147. [CrossRef]

46. Modesto, L.; Rachwal, L. Super-renormalizable and finite gravitational theories. Nucl. Phys. B 2014, 889, 228-248. [CrossRef]

47. Bambi, C.; Modesto, L.; Porey, S.; Rachwal, L. Black hole evaporation in conformal gravity. J. Cosmol. Astropart. Phys. 2017, 2017, 33. [CrossRef]

48. Modesto, L.; Rachwal, L. Finite conformal quantum gravity and spacetime singularities. J. Phys. Conf. Ser. 2017, 942, 012015. [CrossRef]

49. Koshelev, A.S.; Kumar, K.S.; Modesto, L.; Rachwal, L. Finite Quantum Gravity in (A)dS. arXiv 2017, arXiv:1710.07759.

50. Modesto, L.; Rachwal, L.; Shapiro, I.L. Renormalization group in super-renormalizable quantum gravity. arXiv 2017, arXiv:1704.03988.

51. Modesto, L.; Rachwal, L. Finite Quantum Gravity in Four and Extra Dimensions; World Scientific Publishing Co.: Singapore, 2017; doi:10.1142/9789813226609_0084.

52. Modesto, L.; Rachwal, L. Spacetime Singularities in Nonlocal Gravity; World Scientific Publishing Co.: Singapore, 2017; doi:10.1142/9789813226609_0305.

53. Modesto, L.; Rachwal, L. Nonlocal quantum gravity: A review. Int. J. Mod. Phys. D 2017, 26, 1730020. [CrossRef]

54. Codello, A.; Percacci, R.; Rachwal, L.; Tonero, A. Computing the Effective Action with the Functional Renormalization Group. Eur. Phys. J. C 2016, 76, 226. [CrossRef]

55. Rachwal, L.; Codello, A.; Percacci, R. One-Loop Effective Action in Quantum Gravitation. In 1st Karl Schwarzschild Meeting on Gravitational Physics; Springer: Cham, Switzerland, 2016; Volume 170, pp. 395-400.

56. Modesto, L. Super-renormalizable Quantum Gravity. Phys. Rev. D 2012, 86, 044005. [CrossRef]

57. Weinberg, S. Ultraviolet divergences in quantum theories of gravitation. In General Relativity: An Einstein Centenary Survey; Hawking, S.W., Israel, W., Eds.; Cambridge University Press: Cambridge, UK, 1979; pp. 790-831.

58. Reuter, M.; Saueressig, F. Renormalization group flow of quantum gravity in the Einstein-Hilbert truncation. Phys. Rev. D 2002, 65, 065016. [CrossRef]

59. Lauscher, O.; Reuter, M. Is quantum Einstein gravity nonperturbatively renormalizable? Class. Quantum Gravity 2002, 19, 483. [CrossRef] 
60. Litim, D.F. Fixed points of quantum gravity. Phys. Rev. Lett. 2004, 92, 201301. [CrossRef] [PubMed]

61. Kuz'min, Y.V. The Convergent Nonlocal Gravitation. Sov. J. Nucl. Phys. 1989, 50, 1011-1014.

62. Calcagni, G. Multifractional theories: An unconventional review. J. High Energy Phys. 2017, $2017,138$. [CrossRef]

63. Calcagni, G. Towards multifractional calculus. Front. Phys. 2018, 6, 58. [CrossRef]

64. Calcagni, G. Multi-fractional spacetimes, asymptotic safety and Horava-Lifshitz gravity. Int. J. Mod. Phys. A 2013, 28, 1350092. [CrossRef]

65. Biswas, T.; Gerwick, E.; Koivisto, T.; Mazumdar, A. Towards singularity and ghost free theories of gravity. Phys. Rev. Lett. 2012, 108, 031101. [CrossRef] [PubMed]

66. Shapiro, I.L.; Zheksenaev, A.G. Gauge dependence in higher derivative quantum gravity and the conformal anomaly problem. Phys. Lett. B 1994, 324, 286-292. [CrossRef]

67. de Berredo-Peixoto, G.; Shapiro, I.L. Conformal quantum gravity with the Gauss-Bonnet term. Phys. Rev. D 2004, 70, 044024. [CrossRef]

68. Elizalde, E.; Odintsov, S.D.; Romeo, A. Manifestations of quantum gravity in scalar QED phenomena. Phys. Rev. D 1995, 51, 4250. [CrossRef]

69. Elizalde, E.; Odintsov, S.D.; Romeo, A. Improved effective potential in curved space-time and quantum matter, higher derivative gravity theory. Phys. Rev. D 1995, 51, 1680. [CrossRef]

70. Elizalde, E.; Zheksenaev, A.G.; Odintsov, S.D.; Shapiro, I.L. A Four-dimensional theory for quantum gravity with conformal and nonconformal explicit solutions. Class. Quantum Gravity 1995, 12, 1385. [CrossRef]

71. Elizalde, E.; Odintsov, S.D.; Romeo, A. Renormalization group properties of higher derivative quantum gravity with matter in (4-epsilon)-dimensions. Nucl. Phys. B 1996, 462, 315-329. [CrossRef]

72. Codello, A.; D'Odorico, G.; Pagani, C.; Percacci, R. The Renormalization Group and Weyl-invariance. Class. Quantum Gravity 2013, 30, 115015. [CrossRef]

73. Barvinsky, A.O.; Vilkovisky, G.A. The Generalized Schwinger-Dewitt Technique in Gauge Theories and Quantum Gravity. Phys. Rep. 1985, 119, 1-74. [CrossRef]

74. Hollowood, T.J. Renormalization Group and Fixed Points: In Quantum Field Theory; SpringerBriefs in Physics; Springer: Berlin/Heidelberg, Germany, 2013.

75. Johansson, H.; Mogull, G.; Teng, F. Unraveling conformal gravity amplitudes. arXiv 2018, arXiv:1806.05124.

76. Johansson, H.; Nohle, J. Conformal Gravity from Gauge Theory. arXiv 2017, arXiv:1707.02965.

77. Fradkin, E.S.; Tseytlin, A.A. Conformal Supergravity. Phys. Rep. 1985, 119, 233-362. [CrossRef]

78. Fradkin, E.S.; Tseytlin, A.A. Instanton Zero Modes in addition, Beta Functions in Supergravities, Conformal Supergravity. Phys. Lett. B 1984, 134, 307. [CrossRef]

79. Fradkin, E.S.; Tseytlin, A.A. Asymptotic Freedom in Extended Conformal Supergravities. Phys. Lett. B 1982, 110B, 117-122. [CrossRef]

80. Fradkin, E.S.; Tseytlin, A.A. One Loop Beta Function in Conformal Supergravities. Nucl. Phys. B 1982, 203, 157-178. [CrossRef]

81. Giaccari, S.; Modesto, L. Nonlocal supergravity. Phys. Rev. D 2017, 96, 066021. [CrossRef]

82. Cordova, C.; Dumitrescu, T.T.; Intriligator, K. Multiplets of Superconformal Symmetry in Diverse Dimensions. arXiv 2016, arXiv:1612.00809.

83. Bond, A.D.; Litim, D.F.; Vazquez, G.M.; Steudtner, T. UV conformal window for asymptotic safety. Phys. Rev. D 2018, 97, 036019. [CrossRef]

84. t Hooft, G. Local conformal symmetry in black holes, standard model, and quantum gravity. Int. J. Mod. Phys. D 2016, 26, 1730006. [CrossRef]

85. t Hooft, G. Local conformal symmetry: The missing symmetry component for space and time. Int. J. Mod. Phys. D 2015, 24, 1543001. [CrossRef]

86. $\mathrm{t}$ Hooft, G. Spontaneous breakdown of local conformal invariance in quantum gravity. Les Houches Lect. Notes 2015, 97, 209-253.

87. t Hooft, G. Singularities, horizons, firewalls, and local conformal symmetry. arXiv 2015, arXiv:1511.04427.

88. Mannheim, P.D. Making the Case for Conformal Gravity. Found. Phys. 2012, 42, 388-420. [CrossRef]

89. Mannheim, P.D. Mass Generation, the Cosmological Constant Problem, Conformal Symmetry, and the Higgs Boson. Prog. Part. Nucl. Phys. 2017, 94, 125-183. [CrossRef]

90. Mannheim, P.D. Conformal Invariance and the Metrication of the Fundamental Forces. Int. J. Mod. Phys. D 2016, 25, 1644003. [CrossRef] 
91. Jizba, P.; Kleinert, H.; Scardigli, F. Inflationary cosmology from quantum Conformal Gravity. Eur. Phys. J. C 2015, 75, 245. [CrossRef]

92. Zhang, Q.; Modesto, L.; Bambi, C. A general study of regular and singular black hole solutions in Einstein's conformal gravity. Eur. Phys. J. C 2018, 78, 506. [CrossRef]

93. Chakrabarty, H.; Benavides-Gallego, C.A.; Bambi, C.; Modesto, L. Unattainable extended spacetime regions in conformal gravity. J. High Energy Phys. 2018, 2018, 13. [CrossRef]

(C) 2018 by the author. Licensee MDPI, Basel, Switzerland. This article is an open access article distributed under the terms and conditions of the Creative Commons Attribution (CC BY) license (http:/ / creativecommons.org/licenses/by/4.0/). 